\title{
How Do Cannabis Users Mentally Travel in Time: Evidence from an fMRI Study of Episodic Future Thinking
}

\section{Parnian Rafei ${ }^{1}$, Tara Rezapour ${ }^{2}$, Seyed Amir Hossein Batouli ${ }^{3}$, Antonio Verdejo-García ${ }^{4}$, Valentina Lorenzetti ${ }^{5}$, Javad Hatami ${ }^{1}$}

\footnotetext{
${ }^{1}$ Department of Psychology, Faculty of Psychology and Education, University of Tehran, Tehran, Iran

${ }^{2}$ Department of Cognitive Psychology, Institute of Cognitive Sciences Studies (ICSS), Tehran, Iran

${ }^{3}$ Department of Neuroscience and Addiction Studies, School of Advanced Medical Technologies in Medicine, Tehran University of Medical Sciences, Tehran, Iran

${ }^{4}$ Turner Institute for Brain and Mental Health, Monash University, Melbourne, Victoria, Australia

${ }^{5}$ Neuroscience of Addiction and Mental Health Program, Healthy Brain and Mind Research Centre, School of Behavioral \& Health Sciences, Australia Catholic University, Victoria, Australia
}

Corresponding author: Javad Hatami, Hatamijm@ut.ac.ir

\begin{abstract}
:
Background: Episodic Future Thinking (EFT) is a cognitive function that allows individuals to imagine novel experiences that may happen in the future. Prior studies show that EFT is impaired in different groups of substance users such as alcohol and heroin users. However, there is no evidence regarding the neurobiological mechanisms of EFT in cannabis users.

Aim: We aimed to compare brain activations of regular cannabis users and non-using controls during an EFT fMRI task. Exploratory analyses were also conducted to investigate the association between EFT and cannabis use variables (e.g. duration of use, age onset, etc.).
\end{abstract}


Method: Twenty current cannabis-users and 22 drug-naïve controls underwent a single session fMRI while completing a task involving envisioning future-related events and retrieval of past memories as a control condition. The EFT fMRI task was adapted from the autobiographical interview and composed of 20 auditory cue sentences ( 10 cues for past and 10 cues for future events). Participants were asked to recall a past or generate a future event, in response to the cues, and then rated their vividness after each response.

Results: We found that cannabis-users compared to non-user controls had lower activation within the cerebellum, medial, and superior temporal gyrus, lateral occipital cortex, and occipital fusiform gyrus while envisioning future events. Cannabis-users rated the vividness of past events significantly lower than non-users $(\mathrm{P}<0.005)$. There were marginal group differences for rating the vividness of future events $(\mathrm{P}=0.052)$.

Conclusion: Cannabis users, compared to drug-naïve controls, have lower brain activation in EFT relevant regions. Thus, any attempts to improve aberrant EFT performance in cannabis-users may benefit from EFT training.

Keywords: Episodic Future Thinking, Mental Time Travel, Cognitive Functions, Cannabis, fMRI

\section{1) Introduction:}

There has been a significant interest in studying cannabis over the last few years in the field of addiction medicine $(1,2)$. Indeed, cannabis is the most widely used illicit psychoactive substance in the world $(3$, 4). 188 million people worldwide use cannabis (5), and 10 to $30 \%$ of cannabis users endorse symptoms consistent with a Cannabis Use Disorder (CUD) (6). According to the current revision of the Diagnostic and Statistical Manual of Mental Disorders (DSM-5), CUD is defined by criteria including impaired control (e.g., hazardous use), social dysfunctions (e.g., interpersonal problems), risky behaviors, and physiological adaptation (i.e. tolerance) $(7-8,4,9-10)$. Both acute and long-term uses of cannabis are associated with aberrant cognition in a wide range of cognitive domains (11-12). Several neuroimaging 
studies have reported structural (13) and functional (14) brain alterations in individuals with CUD (1517), specifically in the memory-related brain pathways (18) (For a review see 19).

While the association between cannabis use and cognitive functioning has been well-investigated in many cognitive domains (20-23), our knowledge of links between cannabis use and future-oriented cognition is still limited. Episodic Future Thinking (EFT) is a human-specific capacity to mentally simulate the experiences which might happen in one's personal future and to pre-experience novel events (24-27). The Constructive Episodic Simulation Hypothesis proposes that the main process underlying EFT is the episodic memory, which refers to the ability to retrieve and recollect personal events and experiences that happened in the past (28). Neuroimaging findings show that episodic memory, where people retrieve past experiences, and EFT, where people imagine potential future experiences share common neural correlates comprising the Medial Temporal Lobe, Inferior Temporal Gyrus, Precuneus, Posterior Cingulate Cortex, Medial Prefrontal Cortex, and Lateral temporal and Parietal Cortex (29, 27). Results from different studies also associate EFT to a range of higher-order cognitive functions including decision-making, planning, intention formation, and emotion regulation $(30,31)$. Mentally traveling to the future is an essential ability that enables individuals to imagine themselves through different future situations and simulate or predict the actions as well as their consequences and acting upon them (i.e., goal-directed behavior) (32-34). EFT impairment in substance users is clinically significant due to its role in selfcontrol and adaptive behaviors (34-35). Studies show that EFT is a powerful and vital function that guides our actions, based on our imagined future events and their outcomes (36-37). Moreover, in the context of addiction treatment, poor EFT may affect the risk of relapse and exert a significant influence on an individual's response to psychotherapy (38- 39). EFT is an effective facilitation technique to reduce impulsive behaviors and delay discounting, as well as to enhance prospective memory $(40-45,27)$. Therefore, EFT is a critical cognitive function for substance users to mentally imagine the risky situations that may happen in the future and to plan for a goal-based behavioral response (46-47).

In the addiction field, few studies have been probed into EFT function in groups of heroin, alcohol, and cannabis users, as well as pathological gamblers (48-54). Regardless of some methodological differences, 
all these studies point to the existence of impairments in EFT. For example, a study that examined EFT in a group of heroin users concluded that heroin- dependent patients demonstrated a significant deficit in the details for imagined future events (48). Another study concluded that opiate users have difficulties to selfinitiate preparatory behaviors in order to meet their future needs along with a general lack of foresight (49). In another study comparing the EFT between heroin and alcohol users, results showed a significant impairment of EFT associated with heroin use compared to alcohol users and healthy controls (51). Moreover, EFT has been reported to be disrupted in regular cannabis users by generating less episodic details relating to novel future scenarios compared to the groups of recreational users and a non-user group (52). A most recent study found an association between the severity of cannabis use and reduced responsiveness to emotionally intense future events in adolescent cannabis users using an fMRI task (55). However, no previous studies have examined EFT in adult cannabis-users using fMRI, and the existing evidence is mostly originated from findings from behavioral measures.

The rationale behind conducting the present study stems from four key findings of previous studies: a) Episodic memory is the primary function of EFT that is reported to be impaired in cannabis users (56-57, 28); b) The Medial Temporal Lobe (MTL) is the key region of the brain related to EFT, specifically for the scene construction component, which has been indicated to be altered due to heavy use of cannabis $(58-64,29)$; c) EFT deficits are found in psychiatric disorders with common neurobiological substrates as cannabis use, including schizophrenia (65-66) and major depression (67-68); d) Normal function of EFT demands intact executive functions. Meanwhile, the ability to flexibly integrate and recombine the episodic memories related to the past in order to generate, construct, and elaborate future scenarios depends on the executive functions of the brain (69). Therefore, we aimed to examine the neural underpinnings of EFT in cannabis users vs. drug-naïve controls, with a whole-brain analysis approach. Since this study was the first one using fMRI to investigate neural underpinnings of EFT in cannabis users, we used a whole-brain analysis, in an unbiased manner, to not only focus on the brain regions 
formerly known to be involved in EFT, but also other regions that have different activation patterns in cannabis users relative to controls and co-activate while engaging in an EFT task.

We aimed to address the limitation of the literature to date, specifically by: (i) adding neuroimaging evidence, using fMRI as a precise tool, to the limited existing evidence of the relationship between cannabis use and EFT (ii) investigating the association between cannabis use levels (e.g. Frequency of use and the age of onset), and EFT performance (iii) examining the association between global cognitive performance, depression, anxiety, and EFT functioning.

Based on the literature to date, it is hypothesized that (i) cannabis users may show lower brain activations while engaging in an EFT task relative to controls in memory-related brain regions specifically Medial Temporal Lobe as the key region serving EFT (ii) cannabis use patterns such as frequency of use, duration of use, and age of onset may contribute to EFT performance (iii) EFT function in cannabis users may be correlated with levels of depression, anxiety and global cognitive performance.

To achieve these aims, we conducted this fMRI study investigating EFT function in a group of 20 individuals diagnosed with CUD and a control group of 22 people that underwent an fMRI task comprised of EFT and episodic memory conditions.

\section{2) Method}

\section{- Participants}

A total of 42 right-handed adults aged 18 to 37 years were recruited for this study via social media and community advertisements (e.g. flyers). These comprised 20 current cannabis users with a moderate to severe DSM-V CUD, and 22 non-using controls (henceforth termed controls) matched by age, gender, and education level. Participants were screened for: (i) current or prior history of neurological disorders (e.g. multiple sclerosis, epilepsy), head injury with loss of consciousness, traumatic brain injury, (ii) psychiatric disorders (e.g. major depression), (iii) excessive drinking (defined as 21 standard units per week for men and 14 for women) (70), left handedness (measured with 'The Edinburgh handedness 
inventory') (71, 72); (iv) any MRI contraindications; (v) $<25$ score on the Montreal Cognitive Assessment (MoCA) (73-75), (vi) severe depression, i.e. >20 score on the Beck Depression Inventory (BDI-II) (76-77), (vi) severe anxiety, i.e. > 20 score on Beck Anxiety Inventory (BAI) (78). All participants provided written informed consent and were compensated for their participation $(500,000$ IRR). This study was approved by Iran University of Medical Sciences ethics committee (IR.IUMS.REC.1398.070) and was conducted in accordance with the Declaration of Helsinki.

\section{- Screenings and behavioral assessments}

In order to include eligible participants, the following baseline screenings were administered by a trained clinical practitioner:

Depression and anxiety symptom scores were measured by the BDI (76-77) and the BAI (78-79) respectively. CUD was confirmed via using the Persian version of DSM-5 checklist of symptoms (7). MoCa was employed for cognitive impairment screening (cut-off $<25$ ) since it is reported to have an acceptable sensitivity for the rapid identification of cognitive deficits in individuals with substance use disorder $(75,80)$. To tailor the length of cue sentences to participants' memory recall abilities, Rey Auditory Verbal Learning Test (RAVLT) was used (81-82). A six-panel oral fluid (saliva) drug test kit (WONDFO biotech, USA) was employed to screen the participants' drug use (Amphetamines, Methamphetamine, Methadone, Morphine, Benzodiazepine, and Cannabis) and making sure that they are not poly-drug users.

\section{- $\quad$ MRI task}

Figure 1 overviews task trials. We used an fMRI task with two different sets of active trials: a future events generation component that involved envisioning personal situations which might happen in a specific temporal and spatial context in the future, a past retrieval component in which the participants recalled episodic memories related to their past. We used a block design with 2 conditions (10 trials each, total of 20 trials): EFT, episodic memory as an active control, and fixation cross at the beginning of each trial as a passive control. The EFT task was adapted from the Autobiographical Interview (83-84). 
Each trial started with a crosshair fixation with closed eyes (15s), followed by an auditory presented cue sentence (5s), along with a $15 \mathrm{~s}$ gap during which participants had to recall or mentally construct the relevant memory. Immediately after this time, participants were asked to rate the vividness of their memory on a scale from 1 to 4 (ranging from " 1 = inability to imagine or retrieve relevant event" to " $4=$ extremely vivid event") using an MRI-compatible response box placed under their both hands (totally 10s) (Figure 1). Each trial took 45 seconds, and the total duration of the fMRI task was 15 minutes). In both sets of active trials, participants were presented with cue sentences recorded in a female voice, presented in a randomized order using Psychtoolbox-3 software (MATLAB 8.0 and Statistics Toolbox 8.1, The MathWorks, Inc., Natick, Massachusetts, United States) and an MRI-compatible headphone with standard volume.

20 cue sentences that were presented via audio used in the fMRI task. Ten out of 20 cue sentences were related to EFT trials (e.g., "Imagine you are traveling by train in the future"), and the other 10 cue sentences were related to the Episodic memory trials (e.g., "Recall a memory of dining with your family"), The details of the process to select the sentences and normalizing them is described in detail in Supplementary material section 1 .

\section{- Procedures}

Prior to the scanning, participants were provided with verbal instructions and familiarized with the task and did a rehearsal practice outside the scanner, using two cue sentences different from those presented inside the scanner. Due to the importance of visual imagery in EFT (85), all participants were scanned with eyes closed, and all instructions were provided via audio (including presentation of the cue sentences

for EFT and episodic memory conditions as well as instruction for resting state and rating scales). Therefore, any potential effects of visual imagery were not confounded by requirements to visually attend to external task-relevant stimuli (86).

The fMRI task instructions are presented in the Supplementary material section 1. 


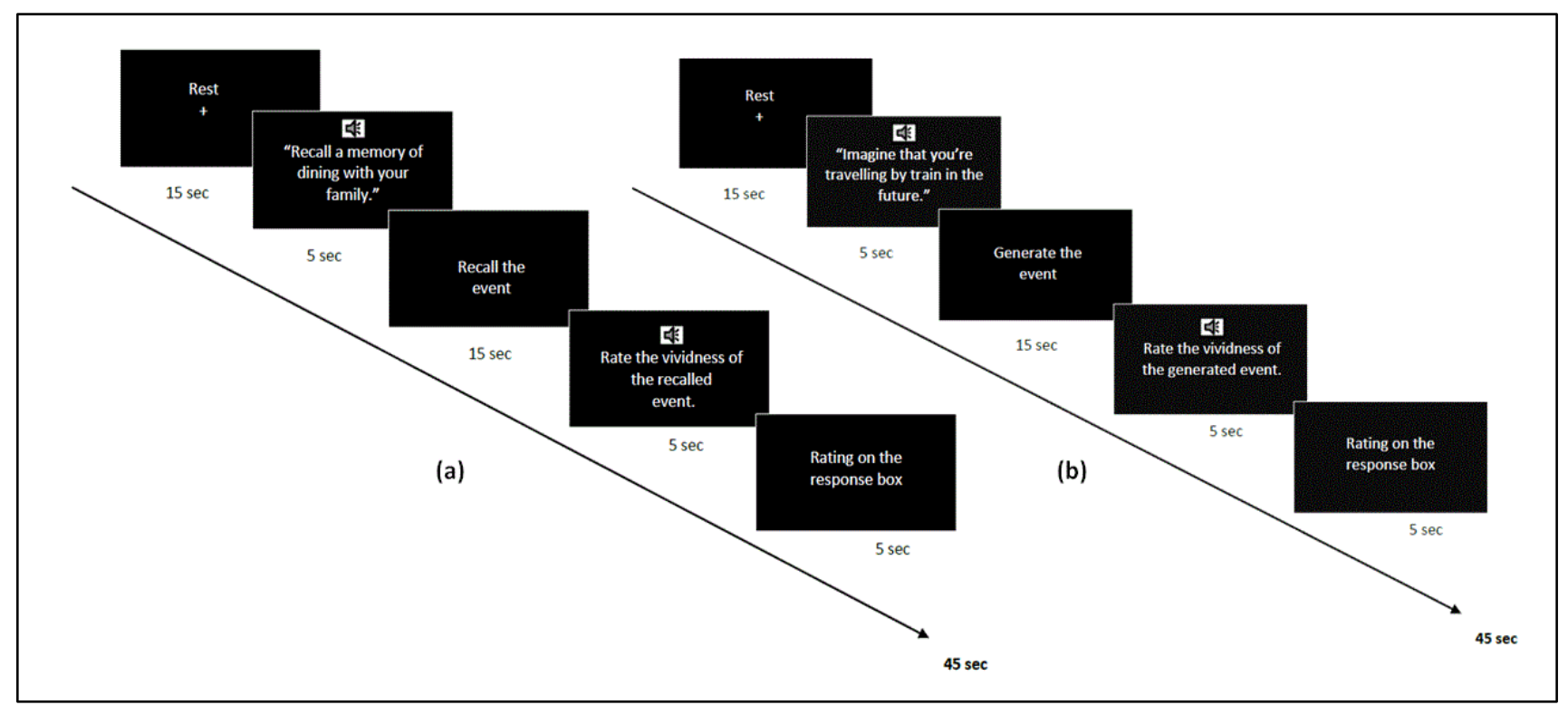

Figure 1: fMRI Experimental Design: a) the episodic memory trial in which participants recalled past events related to the presented cues and rated the vividness of the recalled events; b) The EFT trial in which participants generated future events related to the presented cues and rated the vividness of the generated event

\section{- MRI data acquisition}

Data were acquired on a Siemens MAGNETOM Prisma 3Tesla (Siemens Medical Solutions, Erlangen, Germany) scanner, devoted to research purposes at the National Brain Mapping Lab (www.nbml.ir), using a 64-channel phased-array head coil. At the beginning of the scanning session, standard T1weighted MPRAGE (Magnetization Prepared Rapid Gradient Echo) anatomical scans were obtained $(\mathrm{TA}=4: 12 \mathrm{~min} ; \mathrm{TR}=1800 \mathrm{~ms} ; \mathrm{TE}=3.53 \mathrm{~ms} ; \mathrm{TI}: 1100 \mathrm{~ms} ;$ Voxel size $=1.0 \times 1.0 \times 1.0 \mathrm{~mm} ;$ Flip angle $=7$ degrees; Multi-slice mode $=$ sequential; FOV Read= $256 \mathrm{~mm}$; Matrix size $=256 \times 256 \times 160$; Number of slices $=160$ ). The $\mathrm{T} 2 *$-weighted functional images were acquired using blood oxygen level-dependent (BOLD) contrast, with $40 \mathrm{mT} / \mathrm{m}$ gradients, and by coverage of the whole head and with a gradient-echo echo-planar imaging (EPI) sequence with a repetition time (TR) of $3000 \mathrm{~ms}, 40$ slices with 3.0×3.0×3.0 $\mathrm{mm}$ voxels, an Echo-time (TE) of $30 \mathrm{~ms}$ and a flip angle of 90 degrees. A total of 310 volumes were obtained for a total scanning time length of $930 \mathrm{~s}(15: 30 \mathrm{~m})$. MRI-compatible headphones and response 
keys were employed during the scan. Head Motion was restricted using a standard firm padding that surrounded the head. The headphone volume was set on a comfortable level before starting the scanning session $($ Volume $=4$, Prisma scanner .

\section{- MRI data analysis}

All analyses were performed using the fMRI Expert Analysis Tool (FEAT), part of FMRIB's Software Library (FSL, http://www.fmrib.ox.ac.uk/fsl; 87) (version 5.0.9). The preprocessing procedure consisted of: 1) Motion correction using MCFLIRT, FSL (Motion Correction from FMRIB's Linear Image Registration Tool); 2) Skull-stripping for removal of non-brain tissue from the structural T1-weighted images using Brain Extraction Tool (BET); 3) Slice-timing correction (data acquisition: interleaved); 4) Spatial smoothing using a Gaussian kernel of FWHM= $6.0 \mathrm{~mm}$; 5) Melodic ICA data exploration to identify remaining data artifacts and to help exploring activation in the data; 6) Multiplicative mean intensity normalization of the volume at each time point; 7) High-pass temporal filtering (Gaussianweighted least-squares straight line fitting, with sigma $=60.0 \mathrm{~s}$ ) and; 8) Normalization of the functional images to the standard Montreal Neurological Institute (MNI) (Montreal Neurological Institute, Montreal, Canada) brain atlas was also performed via: I) Co-registration of the functional images to the highresolution T1-weighted scan using FLIRT (FMRIB's Linear Image Registration) and the BBR (Boundary-Based Registration) cost function; and II) Linear registration of the structural T1 images to the MNI space, with 12 DOF.

The first-level analysis was performed using FEAT toolbox, FSL, for each participant, based on a General Linear Model (GLM) statistical analysis. Three regressors were defined in the analysis based on the two active conditions (i.e., EFT and Episodic memory) and the fixation cross control and residuals (e.g., instructions) by creating a boxcar function of tasks (for each condition), against rest by applying the canonical hemodynamic response function. Therefore, for each participant, three images were produced: EFT (future), Episodic memory (past), EFT $>$ EM (future $>$ past). 
Second-level analysis for the group comparisons was performed by FLAME (FMRIB's Local Analysis of Mixed Effects) for calculating the average brain activations of each group, and for measuring the group differences $(Z$-threshold $=>2.3$ ). The false discovery rate was also calculated to control and refine the multiple comparison error $(\mathrm{P}-\mathrm{FDR}<0.05)$.

Anatomic labeling, and locating the activations were performed using the Montreal Neurological Institute (MNI) atlas and Harvard-Oxford cortical and subcortical structural atlases, with a subsequent visual inspection of different activation clusters, overlaid on the T1-weighted image of the MNI152 atlas.

\section{- Behavioral Data Analysis}

The independent samples t-test and $\chi^{2}$ test were used for group comparisons on demographic and substance use levels and other behavioral measures. Pearson correlations were run to explore the associations between demographic and substance use pattern measures with other behavioral results, including BAI, BDI, MoCa, and vividness ratings of past and future events. Statistical analyses were conducted with the SPSS software (version: 19.0, IBM Corp., Armonk, NY, USA, 2017). The P-value was set at $\mathrm{p}<0.05$.

\section{3) Results}

\section{- Demographic characteristics}

Table 1 overviews sample characteristics. 42 Participants aged 21-37 years (Mean= 26.39, SD= 3.59), were eligible for this study. Two participants in the cannabis users group had missing RAVLT data. Groups comprised 22 controls (13 males), and 20 cannabis users (11 males) and were matched by age and years of education (Table 1). The cannabis use pattern of the cannabis user group, along with the alcohol and nicotine use patterns of both groups is demonstrated in Table 1. 
Table 1: Overview of sample' demographic, education, mental health and cognitive characteristics. and alcohol, nicotine and cannabis use patterns

\begin{tabular}{|c|c|c|c|c|c|c|}
\hline & Cannabis users & Controls $(\mathrm{n}=22$ & \multirow[b]{2}{*}{$t(d f)$} & \multirow[b]{2}{*}{$\mathrm{p}$} \\
\hline & & & \multicolumn{2}{|c|}{$\mathrm{N} /$ mean $(\mathrm{SD})$} & & \\
\hline \multicolumn{3}{|c|}{ Gender, total N (male) } & $20(11)$ & $22(13)$ & NA & 0.27 \\
\hline \multicolumn{3}{|l|}{ Age, years } & $26.70(3.32)$ & $26.10(3.88)$ & $-0.53(39)$ & 0.59 \\
\hline \multicolumn{3}{|c|}{ Education, years } & $15.55(1.31)$ & $16.29(1.52)$ & $1.65(39)$ & 0.10 \\
\hline \multicolumn{3}{|l|}{ BAI } & $10.11(7.54)$ & $6.55(5.22)$ & $-1.67(29.86)$ & 0.10 \\
\hline \multicolumn{3}{|l|}{ BDI-II } & $16.11(11.05)$ & $8.55(8.21)$ & $-2.37(31.20)$ & $0.02 *$ \\
\hline \multicolumn{3}{|l|}{ MoCa } & $25.50(1.43)$ & $26.95(1.46)$ & $3.20(38.92)$ & $0.003^{*}$ \\
\hline \multicolumn{3}{|c|}{ RAVLT (Word Span) } & $7.75(1.54)$ & $10.10(1.72)$ & $-3.91(37.64)$ & $0.04 *$ \\
\hline \multicolumn{3}{|c|}{ Past episodes' vividness } & $27.95(3.88)$ & $32.47(4.96)$ & $3.25(37.61)$ & $0.002 *$ \\
\hline \multicolumn{3}{|c|}{ Future episodes' vividness } & $30.75(3.11)$ & $33.43(5.18)$ & $2.01(33.01)$ & 0.052 \\
\hline \multicolumn{3}{|c|}{ Alcohol use (N Yes: No) } & $16: 4$ & $15: 7$ & NA & $0.001^{*}$ \\
\hline \multicolumn{3}{|c|}{ Nicotine use (N Yes: No) } & $14: 6$ & $9: 13$ & NA & 0.27 \\
\hline \multirow[t]{6}{*}{ Cannabis use } & \multicolumn{2}{|c|}{ Age of onset } & $19.35(1.84)$ & NA & NA & NA \\
\hline & \multicolumn{2}{|c|}{ Duration of use (years) } & $7.40(3.05)$ & NA & NA & NA \\
\hline & Frequency & $<2$ & 0 & NA & NA & NA \\
\hline & of use & $3-4$ & 2 & NA & NA & NA \\
\hline & (days per & $5-6$ & 4 & NA & NA & NA \\
\hline & week) & Daily & 14 & NA & NA & NA \\
\hline
\end{tabular}

*P= 〈0.05; NA= not applicable; BAI: Beck Anxiety Inventory; BDI: Beck Depression Inventory; MoCa: Montreal Cognitive Assessment; RAVLT: Ray Auditory Verbal Learning Test 
- Behavioral Results:

\section{a) Baseline assessments group comparison}

Results of the t-test group comparison showed a significant difference in BDI-II and MoCa scores between cannabis user and control groups. There was no significant difference between the two groups in BAI scores, RAVLT word span subset scores of two groups differed significantly as well, but still were in the proposed range (7-9 words) for generating the cue sentences. (Table 1).

\section{b) Episodic future thinking and episodic memory vividness}

The independent samples t-test results have also revealed that the mean of vividness ratings related to past event retrieval trials was significantly different $(\mathrm{t}=3.25, \mathrm{P}<0.005)$ between cannabis user (Mean=27.95, $\mathrm{SD}=3.88$ ) and control groups (Mean=32.47, $\mathrm{SD}=4.96)$. Thus, the vividness ratings of the past episodes were significantly lower in the cannabis users group. The mean of vividness ratings related to the future events construction condition was different $(\mathrm{P}=0.052)$ in the cannabis user $(\mathrm{Mean}=30.75$, $\mathrm{SD}=3.11)$ and control $(33.43, \mathrm{SD}=5.18)$ group comparison, yet not in a statistically significant way.

\section{c) Correlations}

There was a significant negative correlation between mean score of EFT and the level of anxiety (r= $0.52, \mathrm{P}=0.01)$ and the level of depression $(\mathrm{r}=-0.62, \mathrm{P}=0.003)$ in the control group. While, in the cannabis user group, EFT was correlated with the global cognitive functioning (i.e. MoCa scores) $(r=0.49, \mathrm{P}=0.02)$ and the age of onset of cannabis use $(\mathrm{r}=0.50, \mathrm{P}=0.02)$. Furthermore, global cognitive functioning was negatively correlated with the age of onset of cannabis use $(r=-0.68, P=0.001)$. No other correlations were significant. 


\section{- $\quad$ IMRI task results:}

The whole-brain analysis showed that while participants (controls' average activations and cannabisusers' average activations) were engaging in the EFT task and envisioning future events, the Inferior frontal gyrus, Orbitofrontal gyrus, Frontal pole, Insular cortex, Medial frontal gyrus, Frontal operculum cortex, Precuneus, Supracalcarine cortex, Superior parietal lobule, Cerebellum, Occipital fusiform gyrus, Lingual gyrus, Lateral occipital cortex, right Caudate, and Precentral gyrus activations were observed. The EFT>Episodic memory contrast results also revealed significant brain activations in the Medial frontal gyrus, Inferior temporal gyrus, Lingual gyrus, Temporal fusiform gyrus, Lateral occipital cortex, Occipital fusiform gyrus, Precenteral gyrus, right Putamen, and Cerebellum (Table 2 and Figure 2).

The results also showed that while participants were engaging in the episodic memory task and retrieving events that have happened in the past, the Para-hippocampal gyrus, right Hippocampus, Oritofrontal cortex, Medial frontal gyrus, Inferior frontal gyrus, Superior frontal gyrus, Medial temporal gyrus, Inferior temporal gyrus, Postcentral gyrus, Supramarginal gyrus, Para-cingulate gyrus, Lingual gyrus, Post-central gyrus, Supramarginal gyrus, Superior parietal lobule, Occipital fusiform gyrus, Lateral occipital cortex, Angular gyrus, and Cerebellum were activated (Table 2 and Figure 2).

The complete task results are presented in separate tables in supplementary material section 3.

\section{- Group comparisons:}

The whole-brain second-level analysis (i.e. group comparison) compared cannabis users and controls' brain areas activated in EFT and episodic memory trials, comprising in 2 contrasts (cannabisusers $>$ controls, controls $<$ cannabis-users)

Results on brain activations of both groups in the episodic memory and EFT trials are shown in Table 2. Cannabis users vs. controls showed lower cerebellar activations during the EFT task (future contrast) (i.e. left hemisphere, inferior semi-lunar lobule). Moreover, EFT >Episodic memory contrast, which showed the subtraction of past related brain activations from the future condition contrasts of two groups and was our main contrast of interest, indicated a lower brain activation in the cannabis user group vs. controls in 
the medial temporal lobe, superior temporal gyrus, occipital fusiform gyrus, lateral occipital gyrus, and the cerebellum (Figure 2). During the episodic memory trials, the control group demonstrated a higher activation in their right frontal pole and inferior frontal gyrus compared to the cannabis user group, respectively. The cannabis user group did not show any higher brain activations in any of the three experimental conditions, compared to the control group (cannabis-users>controls).

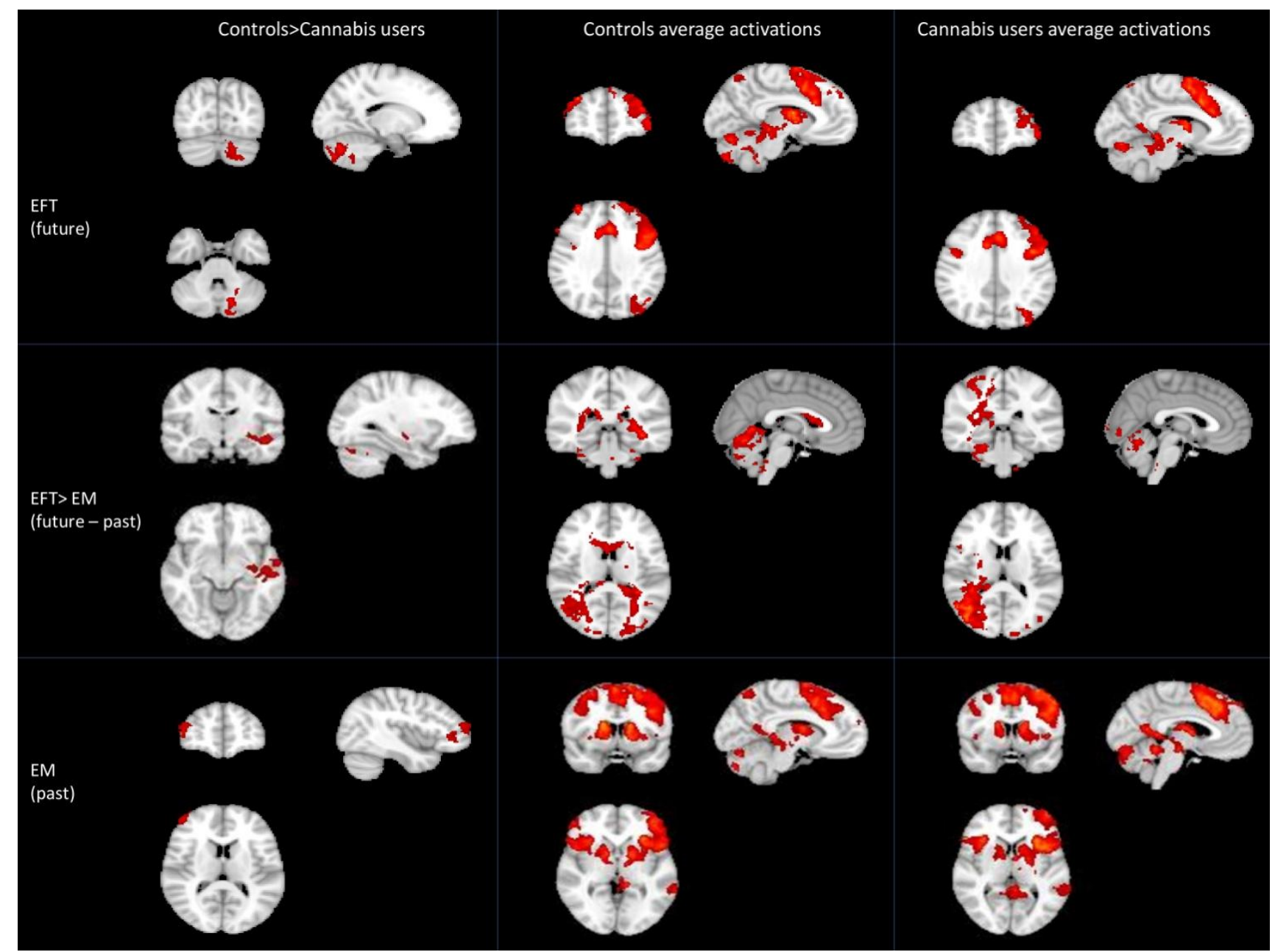

Figure 2. Brain activity patterns associated with 3 experimental conditions (EFT, EFT $>$ Episodic memory, episodic memory), demonstrating 3 contrasts (controls> cannabis-users, controls average activations, and cannabis-users average activations). Zthreshold $=>2.3$

The cannabis user group showed a significant decreased brain activation in the left cerebellum, medial and superior temporal gyrus, occipital fusiform gyrus and the lateral occipital gyrus during the EFT condition, and significant lower brain activation in the right frontal pole and inferior frontal gyrus during the episodic memory condition.

EFT= Episodic Future Thinking, EM=Episodic Memory. 


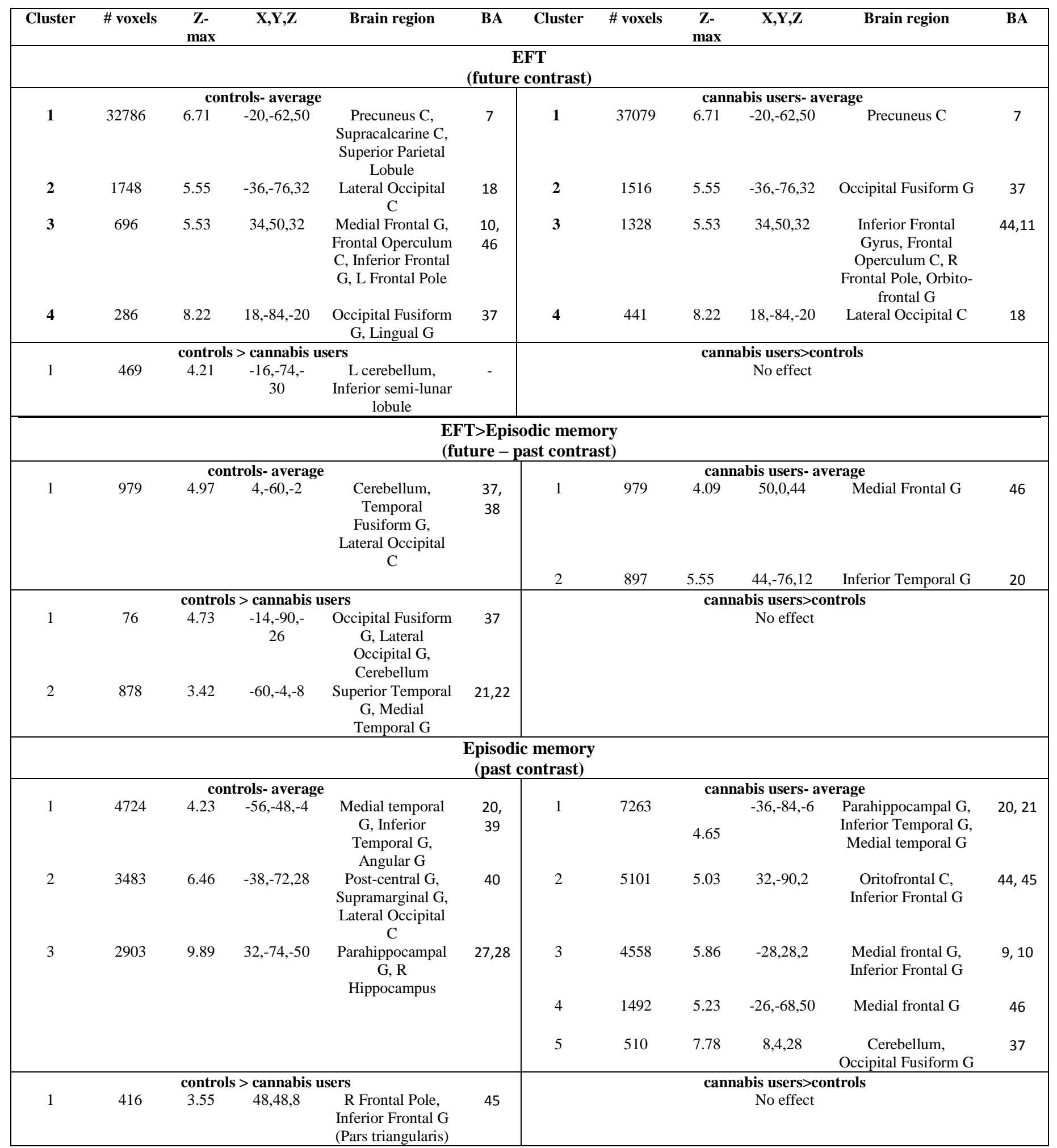

Table 2. The results of brain activations in the EFT and episodic memory conditions as well as EFT>Episodic memory contrast, in the 4 contrasts of cannabis-users>controls, controls < cannabis-users, controls average activations, and cannabis-users average activations

B: bilateral; $R$ : right; L: left; G: gyrus, C: cortex, BA: Brodmann area 
Each activation pattern is reported by the number of clusters, number of voxels in each cluster, maximum z-value of the cluster, and the coordinates $(x, y, z)$ of the voxel with the highest $z$-value in the cluster. The reported clusters survive a whole-brain threshold of $Z>2.3(P<0.05)$.

\section{4) Discussion}

We sought to investigate the brain underpinnings of EFT in cannabis users versus drug-naïve controls. We used fMRI data under two different conditions, including a past retrieval condition in which the participants recalled episodic memories related to their past, as well as a future events generation condition that involved envisioning future personal situations. We found that cannabis users vs. controls have lower brain activations (as measured by BOLD fMRI) in the Medial temporal lobe, Occipital fusiform gyrus, Lateral occipital cortex, and the cerebellum, during an EFT fMRI task; and lower vividness ratings for both past and future events.

Our results extend the prior evidence showing impairment of EFT associated with regular cannabis use adding precise neuroimaging evidence to the sole existing behavioral data (52). Our study, to our best knowledge, is the first study to use fMRI to probe into EFT functioning in cannabis users and compare the brain activations related to envisioning future events to drug-naïve controls using a whole-brain approach, given the exploratory nature of the study.

The function of the Medial Temporal Lobe has been consistently implicated in fMRI studies examining future-oriented cognition (e.g. imagination of hypothetical future scenarios, scene-construction) (88-94, 27). This idea also fits in findings of Medial Temporal Lobe activations during both construction phase of EFT, when individuals initially generate a specific event in response to a cue (e.g., doctor appointment), as well as the elaboration phase, when individuals subsequently elaborate the generated event with as many details (60). There is also existing evidence from a seminal PET study showing significant levels of activations in the Medial temporal lobe as measured by regional cerebral blood flow, while participants were talking about their future prospects, compared to talking about their past experiences (95). Furthermore, the Medial temporal lobe is a core part of the Default Mode Network (96- 99), which has been ascribed to EFT (91, 100-102). Accordingly, the results of our study suggest that cannabis users 
showed decreased activation in the Medial temporal lobe, which may be associated with EFT impairment. Intrestingly, Medial temporal lobe has been reported to be associated with structural (103-106) and functional $(64,107-112)$ brain abnormalities in cannabis users. The Medial temporal lobe plays a major role in the goal-directed decision-making while representing the effects of future on the value of choices (113). This is particularly important in the context of addiction, in which smaller immediate rewards are preferred over larger delayed rewards $(52,114)$.

Our results also show that cannabis users had decreased activation in their cerebellum while imagining themselves in future events. This finding is consistent with that from a previous study showing higher activation of the cerebellum during envisioning future compared to recollecting past experiences (58). Hence, the lower activation in the cerebellum in Cannabis users may potentially account for the lower scores in the subjective vividness ratings of imagined future events in this group. Finally, the cerebellum has a high density of CB1 cannabinoid receptors (115), depicting neuroanatomical and neurofunctional changes in regular Cannabis users compared to non-using controls (116-121, for a review see 122). These findings could potentially justify our results, indicating deficits in cerebellar activity in cannabis users.

In the current study, the Lateral Occipital Cortex has also been found to exhibit lower activation in cannabis users. According to the crucial role of this region in the scene construction element of EFT (123), it could possibly explain the lower scores of cannabis users in subjective vividness ratings of the events. This finding is consistent with the notion that different elements of a recalled or envisioned memory may re-evoke sensory and perceptual information related to the original event $(124,91 ; 29)$. The Lateral Occipital Cortex also plays a critical role in mental imagery and object perception, which are fundamental components of the EFT (125-129).

The Occipital Fusiform Gyrus, which plays an essential role in the visual information processing and face perception (130-131) exhibited lower activations in cannabis users in the current study, while participants were engaged in the EFT task. This region has been shown to contribute to future episodic simulation associated with achieving a goal, as Gerlach and colleagues showed in a group of healthy participants (132). Several fMRI studies have also supported the role of Occipital Fusiform Gyrus in processing the 
visual semantic component of semantic memory (133-136). Notably, semantic memory (i.e. general personal knowledge) is a key component of the EFT (i.e. generate subsequent episodic details) (137-139). Moreover, the Occipital Fusiform Gyrus has a high distribution of CB1 receptors (140) and has been previously reported to have a significantly lower cortical thickness in Cannabis users (141). However, it has been shown that Tetrahydrocannabinol (THC) administration attenuates Occipital Fusiform Gyrus spontaneous activity and evokes visual hallucination in intoxicated individuals (142). Hence, the finding of decreased activation in the Occipital Fusiform Gyrus of cannabis users in the current study may have an important implication in the visual imagery component of EFT, as well as difficulties in generating novel episodes in aid of visual semantic memory in cannabis users.

Our findings also show a significant association between envisioned future events' vividness and global cognitive functioning in cannabis users. Previous studies that assessed global cognitive functioning with MoCA reported significant deficiencies in Cannabis users compared to Controls (143-144). This finding may implicate the notable relationship between EFT and cognitive functions such as memory, which is believed to be serving EFT function as a fundamental component (145). Hence, global cognitive impairment in Cannabis users may potentially reduce their ability to mentally travel in the future.

The other noteworthy association that has been found in this study is the significant relationship between EFT function and the age of onset of cannabis use. We found that those participants who started using cannabis in earlier ages had rated their constructed memories as less vivid in the EFT condition. This result supports the previous literature suggesting the relationship between the age of onset and neurocognitive impairments in this group (146-154).

Our study has several limitations that should be addressed. The first limitation was regarding the baseline assessments that have been done for depression and global cognitive function; although we determined these factors as inclusion and exclusion criteria and recruited those participants who obtained scores in the normal range, a significant difference was found between the groups. Another limitation of this study is the different patterns of substance use among Cannabis users; although the eligible participants 
consisted of those who have not used any drugs except cannabis in the last 60 days, their prior history of substance was relatively diverse.

Nonetheless, to our best knowledge, the current study is among the first studies that used fMRI to measure EFT in substance users. We have provided new evidence for the altered activations in brain regions, including the Medial temporal lobe, Lateral occipital cortex, Occipital fusiform gyrus, and cerebellum in Cannabis users during the EFT condition, and frontal pole and Inferior frontal gyrus during the episodic memory condition. Future studies could benefit from a baseline assessment of participants' mental imagery ability and matching the groups with considering this ability. Additionally, future studies are needed to elucidate the mechanisms of EFT in different substances such as stimulants and psychedelics. Finally, cognitive training programs that apply for substances users (155) may also benefit from targeting EFT (similar to the program used by Ernst et al. (156) and Sofis et al. (157)).

\section{5) Conclusion}

In conclusion, our results provide the first neuroimaging evidence that supports those from previous behavioral studies indicating that cannabis users vs. drug-naïve controls may have difficulties in envisioning the future and acting upon it accordingly. We also found that this impairment affects brain functions in regions associated with episodic future thinking and mental imagery such as the medial temporal lobe, lateral occipital cortex, and cerebellum. These findings have important implications in using and developing new interventions that rely on future-based cognition (simulation, prediction, intention and planning).

\section{6) Acknowledgments}

We thank the National Brain Mapping Lab (NBML) for their excellent services, and Minoo Sisakhti, who kindly assisted with the fMRI task design and data analyses. 


\section{7) Author Disclosure}

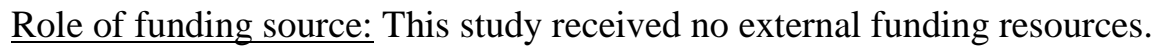

Authors' contribution: PR, TR, JH, SAB, and AVG designed the study. PR managed the literature searches. PR and SAB contributed to fMRI data acquisition and analyses. PR and TR undertook the statistical analysis, and PR, TR, and VL wrote the first draft of the manuscript. All authors contributed to and have approved the final manuscript.

Conflict of Interest: All authors declared no conflict of interest.

\section{8) References}

1) Albright, V. A., \& Johnson, E. O. (2018). Emerging Topics and Innovative Methodologies in Cannabis Research. Substance Abuse: Research and Treatment, 12, 1178221818774264. doi:10.1177/1178221818774264

2) Skibba, R. Why the US decision to expand marijuana supply for research matters. Nature News.

3) National Academies of Sciences, E., \& Medicine. (2017). The health effects of cannabis and cannabinoids: The current state of evidence and recommendations for research: National Academies Press.

4) Volkow, N. D., Baler, R. D., Compton, W. M., \& Weiss, S. R. (2014). Adverse health effects of marijuana use. New England Journal of Medicine, 370(23), 2219-2227.

5) World Health Organization. World Drug Report 2019. United Nations Office on Drugs Crime, 2019 (No. 978, p. 92). Report.

6) Budney, A. J., Sofis, M. J., \& Borodovsky, J. T. (2019). An update on cannabis use disorder with comment on the impact of policy related to therapeutic and recreational cannabis use. European archives of psychiatry and clinical neuroscience, 269(1), 73-86. 
7) American Psychiatric Association. (2013). Diagnostic and statistical manual of mental disorders (DSM-5®). American Psychiatric Pub.

8) Compton, W. M., Dawson, D. A., Goldstein, R. B., \& Grant, B. F. (2013). Crosswalk between DSMIV dependence and DSM-5 substance use disorders for opioids, cannabis, cocaine and alcohol. Drug and alcohol dependence, 132(1-2), 387-390.

9) Hasin, D. S., Kerridge, B. T., Saha, T. D., Huang, B., Pickering, R., Smith, S. M., . . Grant, B. F. (2016). Prevalence and correlates of DSM-5 cannabis use disorder, 2012-2013: Findings from the National Epidemiologic Survey on Alcohol and Related Conditions-III. American Journal of Psychiatry, 173(6), 588-599.

10) Campeny, E., López-Pelayo, H., Nutt, D., Blithikioti, C., Oliveras, C., Nuño, L., . . Gual, A. (2020). The blind men and the elephant: Systematic review of systematic reviews of cannabis use related health harms. European Neuropsychopharmacology, $\quad 33, \quad$ 1-35. doi:https://doi.org/10.1016/j.euroneuro.2020.02.003

11) Solowij N, Pesa N. Cannabis and cognition: short and long-term effects. Marijuana and madness. 2012;2:91-102.

12) Duperrouzel, J. C., Granja, K., Pacheco-Colón, I., \& Gonzalez, R. (2020). Adverse effects of cannabis use on neurocognitive functioning: a systematic review of meta-analytic studies. Journal of Dual Diagnosis, 16(1), 43-57.

13) Lorenzetti, V., Chye, Y., Silva, P., Solowij, N., \& Roberts, C. A. (2019). Does regular cannabis use affect neuroanatomy? An updated systematic review and meta-analysis of structural neuroimaging studies. European archives of psychiatry and clinical neuroscience, 269(1), 59-71.

14) Blest-Hopley, G., Giampietro, V., \& Bhattacharyya, S. (2019). Regular cannabis use is associated with altered activation of central executive and default mode networks even after prolonged abstinence in adolescent users: Results from a complementary meta-analysis. Neuroscience \& Biobehavioral Reviews, 96, 45-55.

15) Nader, D. A., \& Sanchez, Z. M. (2018). Effects of regular cannabis use on neurocognition, brain structure, and function: a systematic review of findings in adults. The American Journal of Drug and Alcohol Abuse, 44(1), 4-18. doi:10.1080/00952990.2017.1306746 
16) Ashton, J. C. (2019). Is Cannabis Harmless? Focus on Brain Function. Current Drug Research Reviews Formerly: Current Drug Abuse Reviews, 11(1), 33-39. doi:10.2174/2589977511666180809165622

17) Burggren, A. C., Shirazi, A., Ginder, N., \& London, E. D. (2019). Cannabis effects on brain structure, function, and cognition: considerations for medical uses of cannabis and its derivatives. The American Journal of Drug and Alcohol Abuse, 45(6), 563-579. doi:10.1080/00952990.2019.1634086

18) Blest-Hopley, G., Giampietro, V., \& Bhattacharyya, S. (2020). A Systematic Review of Human Neuroimaging Evidence of Memory-Related Functional Alterations Associated with Cannabis Use Complemented with Preclinical and Human Evidence of Memory Performance Alterations. Brain Sciences, 10(2), 102.

19) Bloomfield, M. A., Hindocha, C., Green, S. F., Wall, M. B., Lees, R., Petrilli, K., . . Freeman, T. P. (2019). The neuropsychopharmacology of cannabis: a review of human imaging studies. Pharmacology \& therapeutics, 195, 132-161.

20) Broyd, S. J., van Hell, H. H., Beale, C., Yuecel, M., \& Solowij, N. (2016). Acute and chronic effects of cannabinoids on human cognition—a systematic review. Biological psychiatry, 79(7), 557567.

21) Zehra, A., Burns, J., Liu, C. K., Manza, P., Wiers, C. E., Volkow, N. D., \& Wang, G. J. (2018). Cannabis addiction and the brain: a review. Journal of Neuroimmune Pharmacology, 13(4), 438452.

22) Figueiredo, P. R., Tolomeo, S., Steele, J. D., \& Baldacchino, A. (2020). Neurocognitive consequences of chronic cannabis use: A systematic review and meta-analysis. Neuroscience \& Biobehavioral Reviews, 108, 358-369.

23) Lovell, M. E., Akhurst, J., Padgett, C., Garry, M. I., \& Matthews, A. (2019). Cognitive outcomes associated with long-term, regular, recreational cannabis use in adults: A metaanalysis. Experimental and Clinical Psychopharmacology. 
24) Atance, C. M., \& O'Neill, D. K. (2001). Episodic future thinking. Trends in cognitive sciences, 5(12), 533-539.

25) Atance, C. M., \& O’Neill, D. K. (2005). The emergence of episodic future thinking in humans. Learning and Motivation, 36(2), 126-144. doi:https://doi.org/10.1016/j.lmot.2005.02.003

26) Schacter, D. L. (2012). Adaptive constructive processes and the future of memory. American Psychologist, 67(8), 603.

27) Schacter, D. L., Benoit, R. G., \& Szpunar, K. K. (2017). Episodic future thinking: Mechanisms and functions. Current opinion in behavioral sciences, 17, 41-50.

28) Schacter, D. L., \& Addis, D. R. (2007). The cognitive neuroscience of constructive memory: remembering the past and imagining the future. Philosophical Transactions of the Royal Society B: Biological Sciences, 362(1481), 773-786.

29) Weiler, J. A., Suchan, B., \& Daum, I. (2010). When the future becomes the past: Differences in brain activation patterns for episodic memory and episodic future thinking. Behavioural brain research, 212(2), 196-203.

30) D'Argembeau, A., \& Van der Linden, M. (2005). Influence of emotion on memory for temporal information. Emotion, 5(4), 503.

31) Schacter, D. L., Addis, D. R., Hassabis, D., Martin, V. C., Spreng, R. N., \& Szpunar, K. K. (2012). The future of memory: remembering, imagining, and the brain. Neuron, 76(4), 677-694.

32) Suddendorf, T., Addis, D. R., \& Corballis, M. C. (2009). Mental time travel and the shaping of the human mind. Philosophical transactions of the Royal Society of London. Series B, Biological sciences, 364(1521), 1317-1324. doi:10.1098/rstb.2008.0301

33) Suddendorf, T., Brinums, M., \& Imuta, K. (2016). Shaping one's future self-The development of deliberate practice. Seeing the future: Theoretical perspectives on future-oriented mental time travel, 343-366.

34) Bulley, A., \& Irish, M. (2018). The Functions of Prospection - Variations in Health and Disease. Frontiers in Psychology, 9(2328). doi:10.3389/fpsyg.2018.02328 
35) Rezapour, T., Aupperle, R. L., Paulus, M. P., \& Ekhtiari, H. (2020). Clinical translation and implementation neuroscience for novel cognitive interventions in addiction medicine. In Cognition and Addiction (pp. 393-404). Academic Press.

36) Noël, X., Jaafari, N., \& Bechara, A. (2017). Addictive behaviors: Why and how impaired mental time matters?. In Progress in brain research (Vol. 235, pp. 219-237). Elsevier.

37) Jing, H. G., Madore, K. P., \& Schacter, D. L. (2017). Preparing for what might happen: An episodic specificity induction impacts the generation of alternative future events. Cognition, 169, 118-128. doi:10.1016/j.cognition.2017.08.010

38) Van Daele, T., Van den Bergh, O., Van Audenhove, C., Raes, F., \& Hermans, D. (2013). Reduced memory specificity predicts the acquisition of problem solving skills in psychoeducation. Journal of behavior therapy and experimental psychiatry, 44(1), 135-140.

39) Lane, R. D., Ryan, L., Nadel, L., \& Greenberg, L. (2015). Memory reconsolidation, emotional arousal, and the process of change in psychotherapy: New insights from brain science. Behavioral and brain sciences, 38 .

40) Paraskevaides, T., Morgan, C. J., Leitz, J. R., Bisby, J. A., Rendell, P. G., \& Curran, H. V. (2010). Drinking and future thinking: acute effects of alcohol on prospective memory and future simulation. Psychopharmacology, 208(2), 301.

41) Daniel, T. O., Stanton, C. M., \& Epstein, L. H. (2013). The future is now: reducing impulsivity and energy intake using episodic future thinking. Psychological science, 24(11), 2339-2342.

42) Crystal, J. D., \& Wilson, A. G. (2015). Prospective memory: a comparative perspective. Behavioural processes, $112,88-99$.

43) Dassen, F. C., Jansen, A., Nederkoorn, C., \& Houben, K. (2016). Focus on the future: Episodic future thinking reduces discount rate and snacking. Appetite, 96, 327-332.

44) Snider, S. E., LaConte, S. M., \& Bickel, W. K. (2016). Episodic future thinking: Expansion of the temporal window in individuals with alcohol dependence. Alcoholism: Clinical and Experimental Research, 40(7), 1558-1566. 
45) Stein, J. S., Wilson, A. G., Koffarnus, M. N., Daniel, T. O., Epstein, L. H., \& Bickel, W. K. (2016). Unstuck in time: episodic future thinking reduces delay discounting and cigarette smoking. Psychopharmacology, 233(21-22), 3771-3778.

46) Suddendorf, T., \& Corballis, M. C. (2007). The evolution of foresight: What is mental time travel, and is it unique to humans? Behavioral and brain sciences, 30(3), 299-313.

47) Malek, H. B., Berna, F., \& D'Argembeau, A. (2018). Envisioning the times of future events: The role of personal goals. Consciousness and Cognition, 63, 198-205.

48) Mercuri, K., Terrett, G., Henry, J. D., Bailey, P. E., Curran, H. V., \& Rendell, P. G. (2015). Episodic foresight deficits in long-term opiate users. Psychopharmacology, 232(7), 1337-1345.

49) Terrett, G., Lyons, A., Henry, J. D., Ryrie, C., Suddendorf, T., \& Rendell, P. G. (2017). Acting with the future in mind is impaired in long-term opiate users. Psychopharmacology, 234(1), 99-108. doi:10.1007/s00213-016-4442-3

50) Noël, X., Saeremans, M., Kornreich, C., Jaafari, N., \& D'Argembeau, A. (2017). Future-oriented mental time travel in individuals with disordered gambling. Consciousness and cognition, 49, 227-236.

51) Moustafa, A. A., Morris, A. N., Nandrino, J. L., Misiak, B., Szewczuk-Bogusławska, M., Frydecka, D., \& El Haj, M. (2018). Not all drugs are created equal: impaired future thinking in opiate, but not alcohol, users. Experimental Brain Research, 236(11), 2971-2981.

52) Mercuri, K., Terrett, G., Henry, J. D., Curran, H. V., Elliott, M., \& Rendell, P. G. (2018). Episodic foresight deficits in regular, but not recreational, cannabis users. Journal of psychopharmacology, $32(8), 876-882$.

53) Nandrino, J.-L., \& El Haj, M. (2019). The grey future: Overgenerality of emotional future thinking in alcohol-use disorders. Drug and Alcohol Dependence, 205, 107659.

54) El Haj, M., Moustafa, A. A., Perle, A., Tison, P., Cottencin, O., \& Nandrino, J.-L. (2019). Impaired Specificity of Future Thinking in Alcohol Use Disorders. Alcoholism: Clinical and Experimental Research, 43(5), 945-951. doi:10.1111/acer.13993 
55) Aloi, J., Blair, K. S., Meffert, H., White, S. F., Hwang, S., Tyler, P. M., ... \& Filbey, F. M. (2020). Alcohol use disorder and cannabis use disorder symptomatology in adolescents is associated with dysfunction in neural processing of future events. Addiction biology, e12885.

56) Schacter, D. L., Addis, D. R., \& Buckner, R. L. (2008). Episodic simulation of future events: Concepts, data, and applications. Annals of the New York Academy of Sciences, 1124(1), 39-60.

57) Schuster, R. M., Crane, N. A., Mermelstein, R., \& Gonzalez, R. (2015). Tobacco may mask poorer episodic memory among young adult cannabis users. Neuropsychology, 29(5), 759.

58) Szpunar, K. K., Watson, J. M., \& McDermott, K. B. (2007). Neural substrates of envisioning the future. Proceedings of the National Academy of Sciences, 104(2), 642-647. doi:10.1073/pnas.0610082104

59) Botzung, A., Denkova, E., \& Manning, L. (2008). Experiencing past and future personal events: Functional neuroimaging evidence on the neural bases of mental time travel. Brain and cognition, 66(2), 202-212.

60) Schacter, D. L., \& Addis, D. R. (2009). On the nature of medial temporal lobe contributions to the constructive simulation of future events. Philosophical Transactions of the Royal Society B: Biological Sciences, 364(1521), 1245-1253.

61) Addis, D. R., Cheng, T., P. Roberts, R., \& Schacter, D. L. (2011). Hippocampal contributions to the episodic simulation of specific and general future events. Hippocampus, 21(10), 1045-1052.

62) Palombo, D. J., Di Lascio, J. M., Howard, M. W., \& Verfaellie, M. (2019). Medial temporal lobe amnesia is associated with a deficit in recovering temporal context. Journal of cognitive neuroscience, 31(2), 236-248.

63) Yücel, M., Solowij, N., Respondek, C., Whittle, S., Fornito, A., Pantelis, C., \& Lubman, D. I. (2008). Regional brain abnormalities associated with long-term heavy cannabis use. Archives of general psychiatry, 65(6), 694-701. 
64) Ashtari, M., Avants, B., Cyckowski, L., Cervellione, K. L., Roofeh, D., Cook, P., . . Kumra, S. (2011). Medial temporal structures and memory functions in adolescents with heavy cannabis use. Journal of psychiatric research, 45(8), 1055-1066.

65) Rabin, R. A., Goodman, M. S., George, T. P., \& Barr, M. S. (2014). Neurobiology of comorbid substance use disorders in mental illness: A closer look at the underlying commonalities between cannabis and schizophrenia. Current Addiction Reports, 1(4), 261-271.

66) Hallford, D. J., Austin, D. W., Takano, K., \& Raes, F. (2018). Psychopathology and episodic future thinking: A systematic review and meta-analysis of specificity and episodic detail. Behaviour research and therapy, 102, 42-51.

67) Hodgson, K., Almasy, L., Knowles, E. E., Kent Jr, J. W., Curran, J. E., Dyer, T. D., ... \& Fox, P. T. (2017). The genetic basis of the comorbidity between cannabis use and major depression. Addiction, 112(1), 113-123.

68) Moustafa Ahmed, A., Morris Alejandro, N., \& ElHaj, M. (2018). A review on future episodic thinking in mood and anxiety disorders. In Reviews in the Neurosciences (Vol. 30, pp. 85).

69) De Vito, S., Gamboz, N., Brandimonte, M. A., Barone, P., Amboni, M., \& Della Sala, S. (2012). Future thinking in Parkinson's disease: An executive function? Neuropsychologia, 50(7), 14941501. doi:10.1016/j.neuropsychologia.2012.03.001

70) Kalinowski, A., \& Humphreys, K. (2016). Governmental standard drink definitions and low-risk alcohol consumption guidelines in 37 countries. Addiction, 111(7), 1293-1298.

71) Alipour, A., \& Agah Haris, M. (2007). Reliability and validity of Edinburg handedness inventory in Iran. Journal of Psychological Sciences, 22, 117-133.

72) Oldfield, R. C. (1971). The assessment and analysis of handedness: the Edinburgh inventory. Neuropsychologia, 9(1), 97-113.

73) Nasreddine, Z. S., Phillips, N. A., Bédirian, V., Charbonneau, S., Whitehead, V., Collin, I., . . . Chertkow, H. (2005). The Montreal Cognitive Assessment, MoCA: a brief screening tool for mild cognitive impairment. Journal of the American Geriatrics Society, 53(4), 695-699. 
74) Badrkhahan, S. Z., Sikaroodi, H., Sharifi, F., Kouti, L., \& Noroozian, M. (2019). Validity and reliability of the Persian version of the Montreal Cognitive Assessment (MoCA-P) scale among subjects with Parkinson's disease. Appl Neuropsychol Adult, 1-9. doi:10.1080/23279095.2019.1565762

75) Bruijnen, C. J., Jansen, M., Dijkstra, B. A., Walvoort, S. J., Lugtmeijer, S., Markus, W., . . Kessels, R. P. (2019). The Montreal Cognitive Assessment (MoCA) as a cognitive screen in addiction health care: A validation study for clinical practice. Journal of Substance Use, 24(1), 47-54.

76) Beck, A. T., Steer, R. A., \& Brown, G. (1996). Beck depression inventory-II. Psychological Assessment.

77) Ghassemzadeh, H., Mojtabai, R., Karamghadiri, N., \& Ebrahimkhani, N. (2005). Psychometric properties of a Persian-language version of the Beck Depression Inventory-Second edition: BDI-II-PERSIAN. Depression and anxiety, 21(4), 185-192.

78) Wang, Yuan-Pang, \& Gorenstein, Clarice. (2013). Psychometric properties of the Beck Depression Inventory-II: a comprehensive review. Brazilian Journal of Psychiatry, 35(4), 416-431. Epub December 00, 2013.https://doi.org/10.1590/1516-4446-2012-1048

79) Kaviani, H., \& Mousavi, A. (2008). Psychometric properties of the Persian version of Beck Anxiety Inventory (BAI). Tehran University Medical Journal TUMS Publications, 66(2), 136-140.

80) Copersino, M. L., Fals-Stewart, W., Fitzmaurice, G., Schretlen, D. J., Sokoloff, J., \& Weiss, R. D. (2009). Rapid cognitive screening of patients with substance use disorders. Experimental and clinical psychopharmacology, 17(5), 337.

81) Bleecker, M., Ford, D., Lindgren, K., Hoese, V., Walsh, K., \& Vaughan, C. (2005). Differential effects of lead exposure on components of verbal memory. Occupational and environmental medicine, 62(3), 181-187. 
82) Rezvanfard, M., Ekhtiari, H., Noroozian, M., Rezvanifar, A., Nilipour, R., \& KARIMI, J. G. (2011). The Rey Auditory Verbal Learning Test: alternate forms equivalency and reliability for the Iranian adult population (Persian version).

83) Addis, D. R., Wong, A. T., \& Schacter, D. L. (2008). Age-related changes in the episodic simulation of future events. Psychological science, 19(1), 33-41.

84) Levine, B., Svoboda, E., Hay, J. F., Winocur, G., \& Moscovitch, M. (2002). Aging and autobiographical memory: dissociating episodic from semantic retrieval. Psychology and aging, 17(4), 677.

85) De Vito, S., Buonocore, A., Bonnefon, J.-F., \& Della Sala, S. (2015). Eye movements disrupt episodic future thinking. Memory, 23(6), 796-805.

86) Daselaar, S. M., Porat, Y., Huijbers, W., \& Pennartz, C. M. A. (2010). Modality-specific and modality-independent components of the human imagery system. NeuroImage, 52(2), 677-685. doi:https://doi.org/10.1016/j.neuroimage.2010.04.239

87) Smith, S. M., Jenkinson, M., Woolrich, M. W., Beckmann, C. F., Behrens, T. E., Johansen-Berg, H., ... \& Niazy, R. K. (2004). Advances in functional and structural MR image analysis and implementation as FSL. Neuroimage, 23, S208-S219.

88) Race, E., Keane, M. M., \& Verfaellie, M. (2011). Medial temporal lobe damage causes deficits in episodic memory and episodic future thinking not attributable to deficits in narrative construction. Journal of Neuroscience, 31(28), 10262-10269.

89) Benoit, R. G., \& Schacter, D. L. (2015). Specifying the core network supporting episodic simulation and episodic memory by activation likelihood estimation. Neuropsychologia, 75, 450-457.

90) Stawarczyk, D., \& D'Argembeau, A. (2015). Neural correlates of personal goal processing during episodic future thinking and mind-wandering: An ALE meta-analysis. Human Brain Mapping, 36(8), 2928-2947. 
91) Addis, D. R., Pan, L., Vu, M.-A., Laiser, N., \& Schacter, D. L. (2009). Constructive episodic simulation of the future and the past: Distinct subsystems of a core brain network mediate imagining and remembering. Neuropsychologia, 47(11), 2222-2238.

92) Hassabis, D., Kumaran, D., Vann, S. D., \& Maguire, E. A. (2007). Patients with hippocampal amnesia cannot imagine new experiences. Proceedings of the National Academy of Sciences, 104(5), 1726-1731. doi:10.1073/pnas.0610561104

93) Verfaellie, M., Race, E., \& Keane, M. M. (2012). Medial temporal lobe contributions to future thinking: Evidence from neuroimaging and amnesia. Psychologica Belgica, 52(2-3), 77.

94) Palombo, D., Hayes, S., Peterson, K., Keane, M., \& Verfaellie, M. (2018). Medial temporal lobe contributions to episodic future thinking: Scene construction or future projection? Cerebral Cortex, 28(2), 447-458.

95) Okuda, J., Fujii, T., Ohtake, H., Tsukiura, T., Tanji, K., Suzuki, K., . . . Yamadori, A. (2003). Thinking of the future and past: The roles of the frontal pole and the medial temporal lobes. NeuroImage, 19(4), 1369-1380.

96) Raichle, M. E., MacLeod, A. M., Snyder, A. Z., Powers, W. J., Gusnard, D. A., \& Shulman, G. L. (2001). A default mode of brain function. Proceedings of the National Academy of Sciences, 98(2), 676-682.

97) Buckner, R. L., Andrews-Hanna, J. R., \& Schacter, D. L. (2008). The brain's default network: anatomy, function, and relevance to disease.

98) Andrews-Hanna, J. R., Reidler, J. S., Sepulcre, J., Poulin, R., \& Buckner, R. L. (2010). Functionalanatomic fractionation of the brain's default network. Neuron, 65(4), 550-562.

99) Alves, P. N., Foulon, C., Karolis, V., Bzdok, D., Margulies, D. S., Volle, E., \& de Schotten, M. T. (2019). An improved neuroanatomical model of the default-mode network reconciles previous neuroimaging and neuropathological findings. Communications biology, 2(1), 1-14. 
100) Beaty, R. E., Thakral, P. P., Madore, K. P., Benedek, M., \& Schacter, D. L. (2018). Core network contributions to remembering the past, imagining the future, and thinking creatively. Journal of cognitive neuroscience, 30(12), 1939-1951.

101) Bellana, B., Liu, Z. X., Diamond, N., Grady, C., \& Moscovitch, M. (2017). Similarities and differences in the default mode network across rest, retrieval, and future imagining. Human Brain Mapping, 38(3), 1155-1171.

102) Xu, X., Yuan, H., \& Lei, X. (2016). Activation and connectivity within the default mode network contribute independently to future-oriented thought. Scientific reports, 6, 21001.

103) Lorenzetti, V., Batalla, A., \& Cousijn, J. (2016). Cannabis use disorders and altered brain morphology: where is the evidence? Current Addiction Reports, 3(2), 189-198.

104) Lorenzetti, V., Solowij, N., \& Yücel, M. (2016). The role of cannabinoids in neuroanatomic alterations in cannabis users. Biological psychiatry, 79(7), e17-e31.

105) Koenders, L., Cousijn, J., Vingerhoets, W. A., van den Brink, W., Wiers, R. W., Meijer, C. J., . . de Haan, L. (2016). Grey matter changes associated with heavy cannabis use: a longitudinal sMRI study. PloS one, 11(5).

106) Orr, C., Spechler, P., Cao, Z., Albaugh, M., Chaarani, B., Mackey, S., . . Bokde, A. L. (2019). Grey matter volume differences associated with extremely low levels of cannabis use in adolescence. Journal of Neuroscience, 39(10), 1817-1827.

107) Parkar, S. R., Ramanathan, S., Nair, N., Batra, S. A., Adarkar, S. A., Pandit, A. G., . . Baghel, N. S. (2010). Cannabis dependence: Effects of cannabis consumption on inter-regional cerebral metabolic relationships in an Indian population. Indian journal of psychiatry, 52(3), 236.

108) Jacobus, J., Goldenberg, D., Wierenga, C. E., Tolentino, N. J., Liu, T. T., \& Tapert, S. F. (2012). Altered cerebral blood flow and neurocognitive correlates in adolescent cannabis users. Psychopharmacology, 222(4), 675-684. 
109) Riba, J., Valle, M., Sampedro, F., Rodríguez-Pujadas, A., Martínez-Horta, S., Kulisevsky, J., \& Rodríguez-Fornells, A. (2015). Telling true from false: cannabis users show increased susceptibility to false memories. Molecular Psychiatry, 20(6), 772-777.

110) Martin-Santos, R., Fagundo, A. B., Crippa, J. A., Atakan, Z., Bhattacharyya, S., Allen, P., ... \& McGuire, P. (2010). Neuroimaging in cannabis use: a systematic review of the literature. Psychological medicine, 40(3), 383-398.

111) Weinstein, A., Livny, A., \& Weizman, A. (2016). Brain imaging studies on the cognitive, pharmacological and neurobiological effects of cannabis in humans: evidence from studies of adult users. Current pharmaceutical design, 22(42), 6366-6379.

112) Blest-Hopley, G., Giampietro, V., \& Bhattacharyya, S. (2018). Residual effects of cannabis use in adolescent and adult brains-A meta-analysis of fMRI studies. Neuroscience \& Biobehavioral Reviews, 88, 26-41.

113) Palombo, D. J., Keane, M. M., \& Verfaellie, M. (2015). The medial temporal lobes are critical for reward-based decision making under conditions that promote episodic future thinking. Hippocampus, 25(3), 345-353.

114) Grant, S., Contoreggi, C., \& London, E. D. (2000). Drug abusers show impaired performance in a laboratory test of decision making. Neuropsychologia, 38(8), 1180-1187.

115) Glass, M., Faull, R., \& Dragunow, M. (1997). Cannabinoid receptors in the human brain: a detailed anatomical and quantitative autoradiographic study in the fetal, neonatal and adult human brain. Neuroscience, 77(2), 299-318.

116) Medina, K. L., Nagel, B. J., \& Tapert, S. F. (2010). Abnormal cerebellar morphometry in abstinent adolescent marijuana users. Psychiatry Research: Neuroimaging, 182(2), 152-159.

117) Cousijn, J., Wiers, R. W., Ridderinkhof, K. R., van den Brink, W., Veltman, D. J., \& Goudriaan, A. E. (2012). Grey matter alterations associated with cannabis use: Results of a VBM study in heavy cannabis users and healthy controls. NeuroImage, 59(4), 3845-3851. doi:https://doi.org/10.1016/j.neuroimage.2011.09.046 
118) Sweigert, J., Pagulayan, K., Greco, G., Blake, M., Larimer, M., \& Kleinhans, N. M. (2019). A multimodal investigation of cerebellar integrity associated with high-risk cannabis use. Addiction Biology, e12839.

119) Batalla, A., Bhattacharyya, S., Yuecel, M., Fusar-Poli, P., Crippa, J. A., Nogue, S., . . . MartinSantos, R. (2013). Structural and functional imaging studies in chronic cannabis users: a systematic review of adolescent and adult findings. PloS one, 8(2).

120) Wetherill, R. R., Fang, Z., Jagannathan, K., Childress, A. R., Rao, H., \& Franklin, T. R. (2015). Cannabis, cigarettes, and their co-occurring use: Disentangling differences in default mode network functional connectivity. Drug and Alcohol Dependence, 153, 116-123.

121) Lorenzetti, V., Chye, Y., Suo, C., Walterfang, M., Lubman, D. I., Takagi, M., . . Pantelis, C. (2020). Neuroanatomical alterations in people with high and low cannabis dependence. Australian \& New Zealand Journal of Psychiatry, 54(1), 68-75.

122) Moreno-Rius, J. (2019). The Cerebellum, THC, and Cannabis Addiction: Findings from Animal and Human Studies. The Cerebellum, 1-12.

123) Summerfield, J. J., Hassabis, D., \& Maguire, E. A. (2010). Differential engagement of brain regions within a 'core'network during scene construction. Neuropsychologia, 48(5), 1501-1509.

124) Johnson, M. K., Foley, M. A., Suengas, A. G., \& Raye, C. L. (1988). Phenomenal characteristics of memories for perceived and imagined autobiographical events. Journal of Experimental Psychology: General, 117(4), 371.

125) D'Esposito, M., Detre, J. A., Aguirre, G. K., Stallcup, M., Alsop, D. C., Tippet, L. J., \& Farah, M. J. (1997). A functional MRI study of mental image generation. Neuropsychologia, 35(5), 725-730.

126) Grill-Spector, K., Kourtzi, Z., \& Kanwisher, N. (2001). The lateral occipital complex and its role in object recognition. Vision research, 41(10-11), 1409-1422.

127) Kosslyn, S. M., \& Thompson, W. L. (2003). When is early visual cortex activated during visual mental imagery? Psychol Bull, 129(5), 723-746. doi:10.1037/0033-2909.129.5.723 
128) Moulton, S. T., \& Kosslyn, S. M. (2009). Imagining predictions: mental imagery as mental emulation. Philosophical Transactions of the Royal Society B: Biological Sciences, 364(1521), 1273-1280.

129) Cichy, R. M., Heinzle, J., \& Haynes, J.-D. (2012). Imagery and perception share cortical representations of content and location. Cerebral Cortex, 22(2), 372-380.

130) Kanwisher, N., McDermott, J., \& Chun, M. M. (1997). The fusiform face area: a module in human extrastriate cortex specialized for face perception. Journal of Neuroscience, 17(11), 4302-4311.

131) Weiner, K. S., \& Zilles, K. (2016). The anatomical and functional specialization of the fusiform gyrus. Neuropsychologia, 83, 48-62.

132) Gerlach, K. D., Spreng, R. N., Madore, K. P., \& Schacter, D. L. (2014). Future planning: default network activity couples with frontoparietal control network and reward-processing regions during process and outcome simulations. Social cognitive and affective neuroscience, 9(12), 1942-1951.

133) Mion, M., Patterson, K., Acosta-Cabronero, J., Pengas, G., Izquierdo-Garcia, D., Hong, Y. T., . . . Nestor, P. J. (2010). What the left and right anterior fusiform gyri tell us about semantic memory. Brain, 133(11), 3256-3268. doi:10.1093/brain/awq272

134) Sabsevitz, D. S., Medler, D. A., Seidenberg, M., \& Binder, J. R. (2005). Modulation of the semantic system by word imageability. NeuroImage, 27(1), 188-200. doi:https://doi.org/10.1016/j.neuroimage.2005.04.012

135) Pins, D., Meyer, M.-E., Foucher, J., Humphreys, G., \& Boucart, M. (2004). Neural correlates of implicit object identification. Neuropsychologia, 42(9), 1247-1259.

136) Simons, J. S., Koutstaal, W., Prince, S., Wagner, A. D., \& Schacter, D. L. (2003). Neural mechanisms of visual object priming: evidence for perceptual and semantic distinctions in fusiform cortex. NeuroImage, 19(3), 613-626.

137) D'Argembeau, A., \& Mathy, A. (2011). Tracking the construction of episodic future thoughts. Journal of Experimental Psychology: General, 140(2), 258. 
138) Irish, M., \& Piolino, P. (2016). Impaired capacity for prospection in the dementias-Theoretical and clinical implications. British Journal of Clinical Psychology, 55(1), 49-68.

139) Irish, M., Eyre, N., Dermody, N., O’Callaghan, C., Hodges, J. R., Hornberger, M., \& Piguet, O. (2016). Neural substrates of semantic prospection-Evidence from the dementias. Frontiers in Behavioral Neuroscience, 10, 96.

140) Wong, D. F., Kuwabara, H., Horti, A. G., Raymont, V., Brasic, J., Guevara, M., . . Nandi, A. (2010). Quantification of cerebral cannabinoid receptors subtype 1 (CB1) in healthy subjects and schizophrenia by the novel PET radioligand [11C] OMAR. NeuroImage, 52(4), 1505-1513.

141) Mashhoon, Y., Sava, S., Sneider, J., Nickerson, L., \& Silveri, M. (2015). Cortical thinness and volume differences associated with marijuana abuse in emerging adults. Drug and Alcohol Dependence, 155, 275-283.

142) Winton-Brown, T. T., Allen, P., Bhattacharrya, S., Borgwardt, S. J., Fusar-Poli, P., Crippa, J. A., . . . Zuardi, A. W. (2011). Modulation of auditory and visual processing by delta-9tetrahydrocannabinol and cannabidiol: an FMRI study. Neuropsychopharmacology, 36(7), 13401348.

143) Cengel, H. Y., Bozkurt, M., Evren, C., Umut, G., Keskinkilic, C., \& Agachanli, R. (2018). Evaluation of cognitive functions in individuals with synthetic cannabinoid use disorder and comparison to individuals with cannabis use disorder. Psychiatry research, 262, 46-54.

144) Umut, G., Atagun, M. I., Evren, C., Cengel, H. Y., Hisim, O., \& Keskinkilic, C. (2016). The changes of the cognitive and psychomotor functions in the chronic cannabis users after a month of remission. Klinik Psikofarmakoloji Bülteni-Bulletin of Clinical Psychopharmacology, 26(2), 109118.

145) Irish, M., Addis, D. R., Hodges, J. R., \& Piguet, O. (2012). Considering the role of semantic memory in episodic future thinking: evidence from semantic dementia. Brain, 135(7), 2178-2191. 
146) Pope, H. G., Gruber, A. J., Hudson, J. I., Cohane, G., Huestis, M. A., \& Yurgelun-Todd, D. (2003). Early-onset cannabis use and cognitive deficits: what is the nature of the association? Drug and Alcohol Dependence, 69(3), 303-310. doi:https://doi.org/10.1016/S0376-8716(02)00334-4

147) Jager, G., \& Ramsey, N. F. (2008). Long-term consequences of adolescent cannabis exposure on the development of cognition, brain structure and function: an overview of animal and human research. Current drug abuse reviews, 1(2), 114-123.

148) Becker, B., Wagner, D., Gouzoulis-Mayfrank, E., Spuentrup, E., \& Daumann, J. (2010). The impact of early-onset cannabis use on functional brain correlates of working memory. Progress in Neuro-Psychopharmacology and Biological Psychiatry, 34(6), 837-845.

149) Fontes, M. A., Bolla, K. I., Cunha, P. J., Almeida, P. P., Jungerman, F., Laranjeira, R. R., . . . Lacerda, A. L. (2011). Cannabis use before age 15 and subsequent executive functioning. The British Journal of Psychiatry, 198(6), 442-447.

150) Volkow, N. D., Swanson, J. M., Evins, A. E., DeLisi, L. E., Meier, M. H., Gonzalez, R., . . Baler, R. (2016). Effects of cannabis use on human behavior, including cognition, motivation, and psychosis: a review. JAMA Psychiatry, 73(3), 292-297.

151) Gruber, S. A., Sagar, K. A., Dahlgren, M. K., Racine, M., \& Lukas, S. E. (2012). Age of onset of marijuana use and executive function. Psychology of Addictive Behaviors, 26(3), 496.

152) Lubman, D. I., Cheetham, A., \& Yücel, M. (2015). Cannabis and adolescent brain development. Pharmacology \& therapeutics, 148, 1-16.

153) Gorey, C., Kuhns, L., Smaragdi, E., Kroon, E., \& Cousijn, J. (2019). Age-related differences in the impact of cannabis use on the brain and cognition: a systematic review. European archives of psychiatry and clinical neuroscience, 269(1), 37-58.

154) Harvey, P. D. (2019). Smoking cannabis and acquired impairments in cognition: starting early seems like a really bad idea. In: Am Psychiatric Assoc. 
155) Rezapour, T., DeVito, E. E., Sofuoglu, M., \& Ekhtiari, H. (2016). Perspectives on neurocognitive rehabilitation as an adjunct treatment for addictive disorders: from cognitive improvement to relapse prevention. In Progress in brain research (Vol. 224, pp. 345-369). Elsevier.

156) Ernst, A., Sourty, M., Roquet, D., Noblet, V., Gounot, D., Blanc, F., . . . Manning, L. (2016). Functional and structural cerebral changes in key brain regions after a facilitation programme for episodic future thought in relapsing-remitting multiple sclerosis patients. Brain and cognition, 105, 34-45. doi:https://doi.org/10.1016/j.bandc.2016.03.007

157) Sofis, M. J., Lemley, S. M., Lee, D. C., \& Budney, A. J. (2020). A web-based episodic specificity and future thinking session modulates delay discounting in cannabis users. Psychology of Addictive Behaviors, 34(4), 532.

\section{- Supplementary materials- section 1}

The adapted cue words were checked for concreteness and imageability and then transformed into short sentences (Clark \& Paivio, 1991). According to the participants memory abilities (assessed by RAVLT word span subtest), cue sentences, including 7-9 words, were developed. The developed sentences were subsequently normalized in a group of 40 healthy subjects to be rated for imageability and familiarity (having previous experience of the situation) on a 4- point Likert scale (ranging from "1 = very hard to imagine" to " 4 = clearly imaginable"). Eventually, 20 cue sentences were selected to be presented in the fMRI scanner. The selected cue sentences for EFT condition were rated highly imaginable among $80 \%$ $(n=32)$ of the raters, and the cues selected for episodic memory condition were indicated to have been formerly experienced among $90 \%(n=36)$ of the raters. Ten out of 20 cue sentences were related to the episodic memory condition to be retrieved (e.g., "Recall a memory of dining with your family"), and the other 10 cue sentences were related to EFT condition to be envisioned (e.g., "Imagine you are traveling by train in the future").

\section{- Supplementary materials- section 2}

The given instructions for the EFT condition was as follows: "You will hear a short sentence proposing you to imagine a novel event happening in your future; after hearing the sentence, you will have 15 seconds to generate and imagine the event happening in a specific time and place as an episode of a movie in the first-person point of view, then you have to appraise the vividness of the event that you imagined, based on the details you have constructed as imagining the future event and rate it on a 1 to 4 scale, at last". For the episodic memory condition, participants were instructed as follows "You will hear a short sentence proposing you to recall a previously happened event in your past (i.e., a memory); after hearing the sentence, you will have 15 seconds to recall the event that has happened in a specific time and place as an episode of a movie in the first-person point of view, then you have to appraise the 
vividness of the event that you recalled, based on the details you have remembered as recalling the memory and rate it on a 1 to 4 scale, at last".

- Supplementary materials- section 3

\section{fMRI task results:}

\section{1) Contrast 1- EFT (future)}

a) Controls:

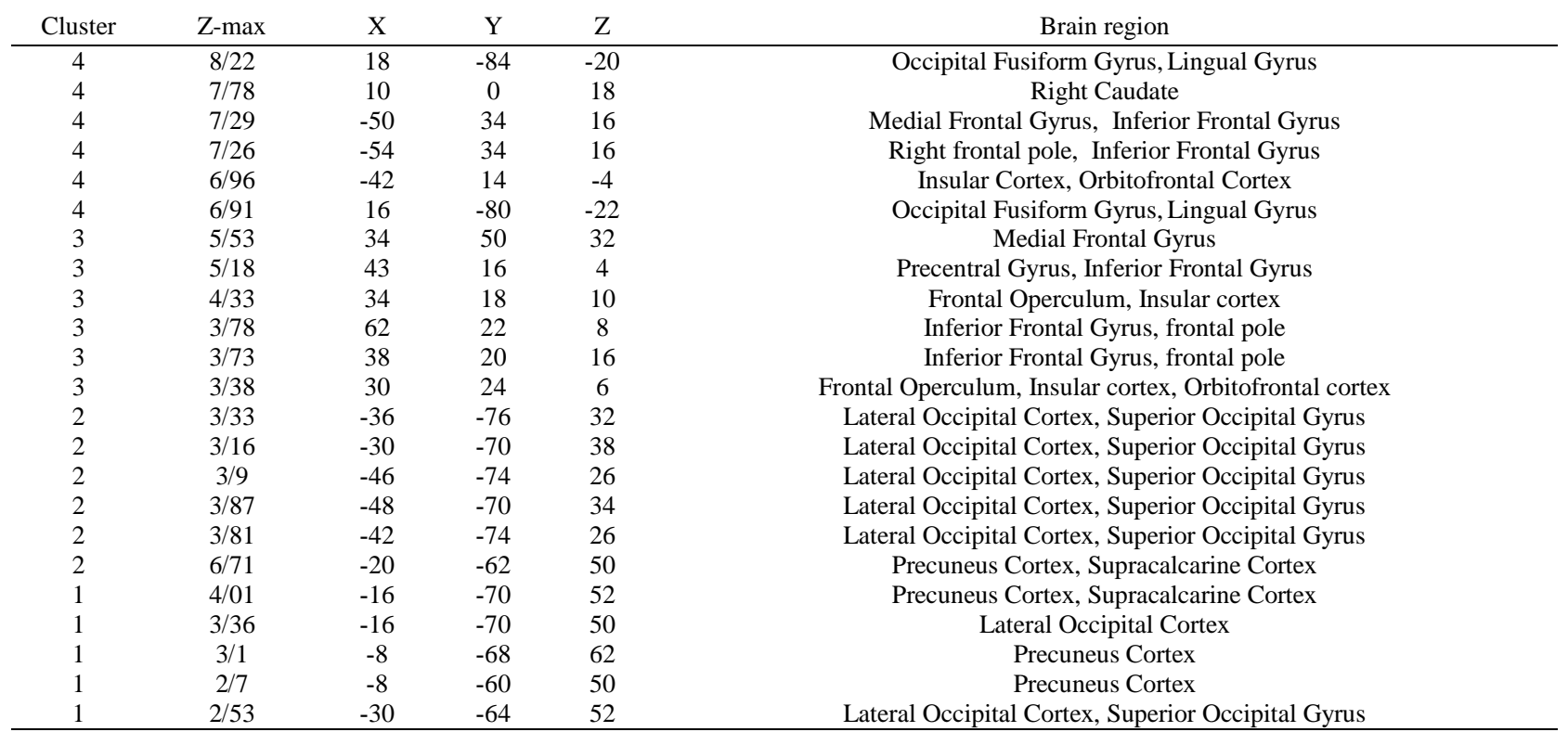

The results of brain activations in the EFT contrast- controls' average activations

Each activation pattern is reported by the number of clusters, number of voxels in each cluster, maximum z-value of the cluster, and the coordinates $(x, y, z)$ of the voxel with the highest $z$-value in the cluster. The reported clusters survive a whole-brain threshold of $Z>2.3(P<0.05)$. 


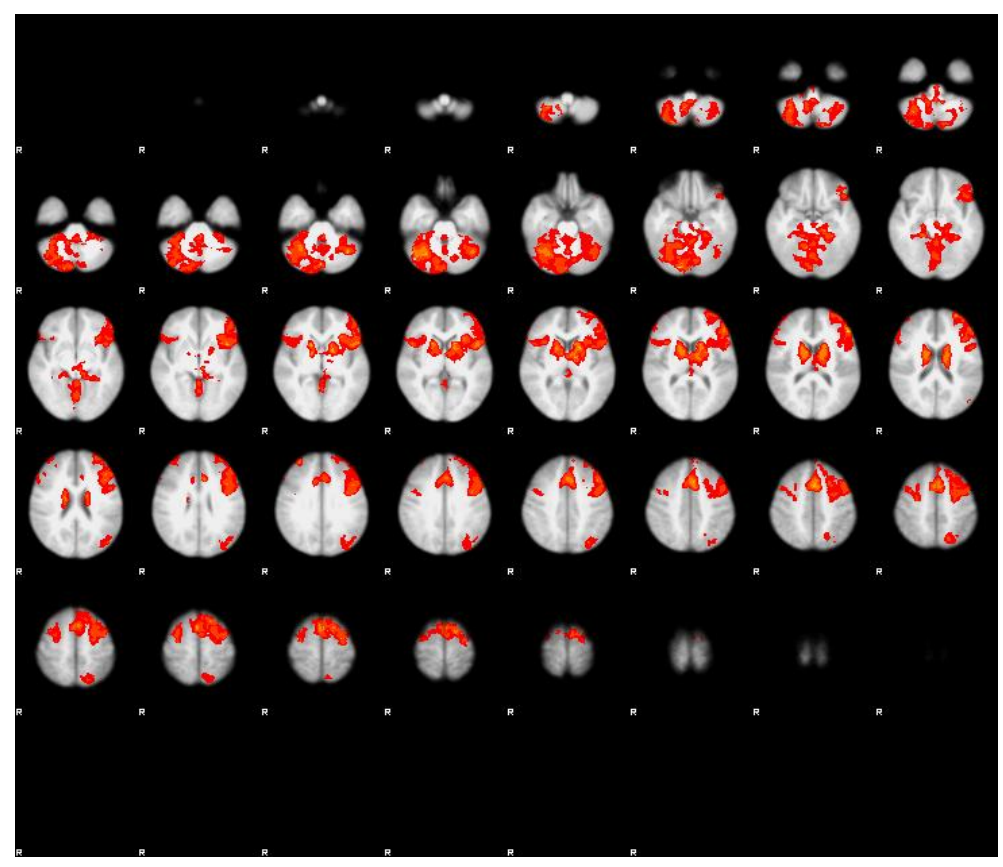

Brain activity patterns associated with EFT condition in drug-naïve controls (Z-threshold= >2.3)

b) Cannabis users

\begin{tabular}{|c|c|c|c|c|c|}
\hline Cluster & Z-max & $\mathrm{X}$ & $\mathrm{Y}$ & $\mathrm{Z}$ & Brain region \\
\hline 4 & $8 / 22$ & 18 & -84 & -20 & Lateral Occipital Cortex \\
\hline 4 & 7/78 & 10 & 0 & 18 & Right Caudate \\
\hline 4 & $7 / 29$ & -50 & 34 & 16 & Inferior Frontal Gyrus \\
\hline 4 & $7 / 26$ & -54 & 34 & 16 & Inferior Frontal Gyrus \\
\hline 4 & $6 / 96$ & -42 & 14 & -4 & Insular cortex \\
\hline 4 & $6 / 91$ & 16 & -80 & -22 & Occipital Fusiform Gyrus \\
\hline 3 & $5 / 53$ & 34 & 50 & 32 & Frontal pole \\
\hline 3 & $5 / 18$ & 48 & 16 & 4 & Inferior Frontal Gyrus \\
\hline 3 & $4 / 33$ & 34 & 18 & 10 & Insular cortex \\
\hline 3 & $3 / 78$ & 62 & 22 & 8 & Inferior Frontal Gyrus \\
\hline 3 & $3 / 73$ & 58 & 20 & 16 & Inferior Frontal Gyrus \\
\hline 3 & $3 / 58$ & 30 & 24 & 6 & Orbitofrontal Cortex \\
\hline 2 & $5 / 55$ & -36 & -76 & 32 & Lateral Occipital Cortex \\
\hline 2 & $5 / 16$ & -30 & -70 & 38 & Lateral Occipital Cortex \\
\hline 2 & $5 / 16$ & -30 & -74 & 40 & Lateral Occipital Cortex \\
\hline 2 & $3 / 9$ & -46 & -74 & 26 & Lateral Occipital Cortex \\
\hline 2 & $3 / 87$ & -48 & -70 & 34 & Lateral Occipital Cortex \\
\hline 2 & $3 / 81$ & -42 & -74 & 26 & Lateral Occipital Cortex \\
\hline 1 & $6 / 71$ & -20 & -62 & 50 & Precuneus Cortex \\
\hline 1 & $4 / 01$ & -16 & -70 & 52 & Precuneus Cortex \\
\hline 1 & $3 / 36$ & -16 & -72 & 60 & Precuneus Cortex \\
\hline 1 & $3 / 1$ & -8 & -68 & 62 & Precuneus Cortex \\
\hline 1 & $2 / 7$ & -8 & -60 & 60 & Precuneus Cortex \\
\hline 1 & $2 / 53$ & -30 & -64 & 52 & Precuneus Cortex, Lateral Occipital Cortex \\
\hline
\end{tabular}

The results of brain activations in the EFT contrast- cannabis users' average activations

Each activation pattern is reported by the number of clusters, number of voxels in each cluster, maximum z-value of the cluster, and the coordinates $(x, y, z)$ of the voxel with the highest $z$-value in the cluster. The reported clusters survive a whole-brain threshold of $Z>2.3(P<0.05)$. 


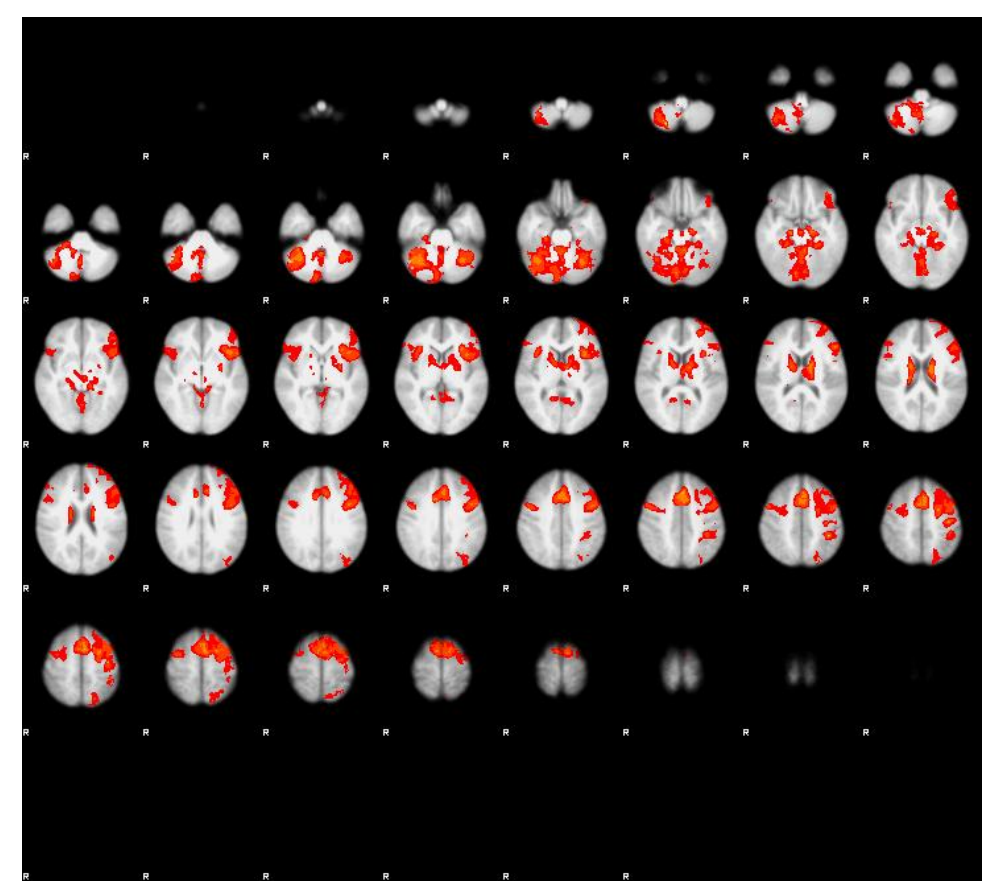

Brain activity patterns associated with EFT condition in cannabis users (Z-threshold $=>2.3$ )

c) Contols>cannabis users

\begin{tabular}{cccccc} 
Cluster & Z-max & $\mathrm{X}$ & $\mathrm{Y}$ & $\mathrm{Z}$ & Brain region \\
\hline 1 & $4 / 21$ & -16 & -74 & -30 & Left Cerebellum- Inferior Semi-lunar lobule \\
1 & $4 / 09$ & -18 & -58 & -44 & Left Cerebellum- Inferior Semi-lunar lobule \\
1 & $4 / 01$ & -26 & -80 & -46 & Left Cerebellum- Inferior Semi-lunar lobule \\
1 & 4 & -18 & -80 & -44 & Left Cerebellum \\
1 & $3 / 5$ & -12 & -86 & -32 & Left Cerebellum \\
1 & $3 / 38$ & -24 & -72 & -44 & Left Cerebellum \\
\hline
\end{tabular}

The results of brain activations in the EFT contrast- Contols>cannabis users

Each activation pattern is reported by the number of clusters, number of voxels in each cluster, maximum z-value of the cluster, and the coordinates $(x, y, z)$ of the voxel with the highest $z$-value in the cluster. The reported clusters survive a whole-brain threshold of $Z>2.3(P<0.05)$. 


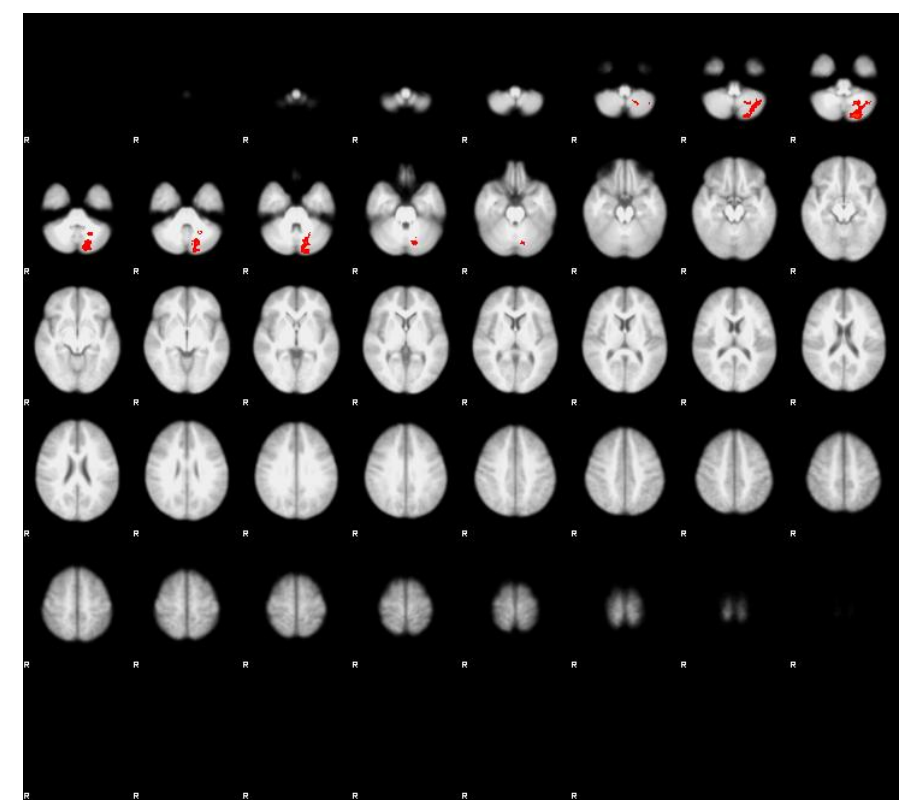

Brain activity differences associated with EFT condition in control $>$ cannabis user contrast $($ Z-threshold $=>2.3$ )

\section{2) Contrast 2- EFT > Episodic memory}

a) Controls:

\begin{tabular}{|c|c|c|c|c|c|}
\hline Cluster & Z-max & $\mathrm{X}$ & $\mathrm{Y}$ & $\mathrm{Z}$ & Brain region \\
\hline 1 & $4 / 97$ & 4 & -60 & -2 & Lingual Gyrus, Cerebellum \\
\hline 1 & $4 / 86$ & 4 & -66 & -14 & Cerebellum \\
\hline 1 & $4 / 82$ & 8 & -64 & -12 & Lingual Gyrus, Cerebellum \\
\hline 1 & $4 / 77$ & -36 & -32 & -8 & Temporal Fusiform Gyrus \\
\hline 1 & $4 / 62$ & -4 & -74 & -12 & Occipital Fusiform Gyrus \\
\hline 1 & $4 / 61$ & 40 & -78 & 2 & Lateral Occipital Cortex \\
\hline
\end{tabular}

The results of brain activations in the EFT $>$ Episodic memory contrast- controls' average activations

Each activation pattern is reported by the number of clusters, number of voxels in each cluster, maximum z-value of the cluster, and the coordinates $(x, y, z)$ of the voxel with the highest $z$-value in the cluster. The reported clusters survive a whole-brain threshold of $Z>2.3(P<0.05)$. 


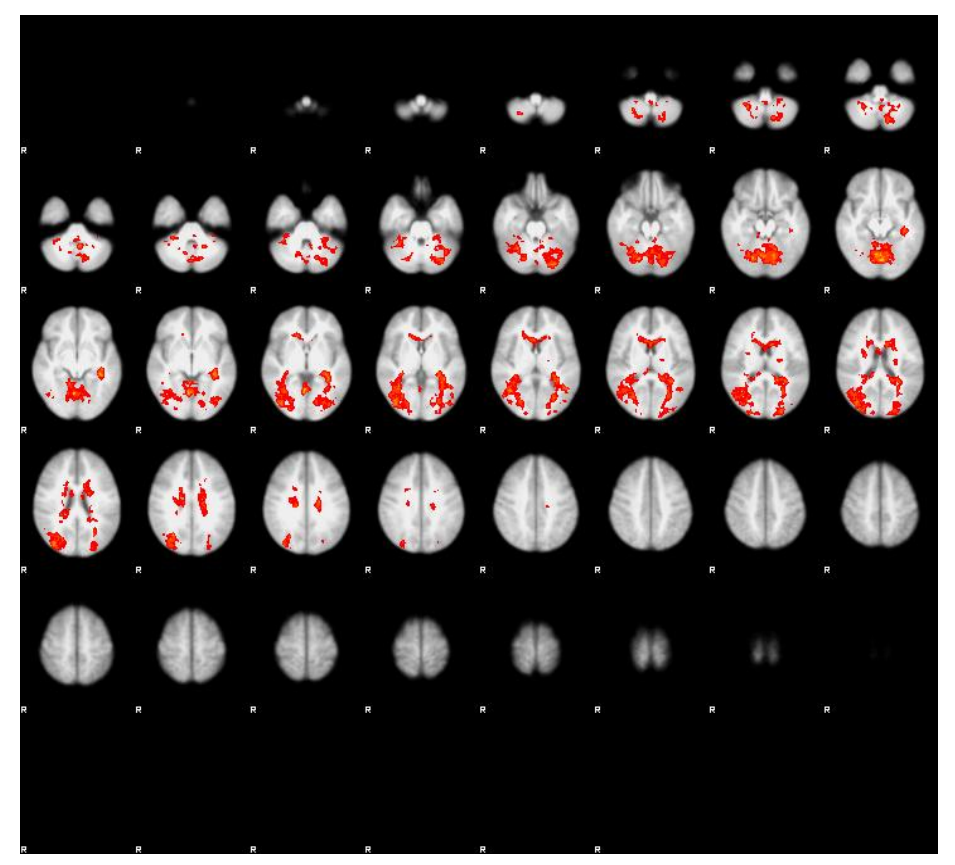

Brain activity patterns associated with EFT $>$ EM contrast in drug-naïve controls $(\mathrm{Z}$-threshold= $>2.3$ )

b) Cannabis users:

\begin{tabular}{cccccc} 
Cluster & Z-max & $\mathrm{X}$ & $\mathrm{Y}$ & $\mathrm{Z}$ & Brain region \\
\hline 2 & $5 / 55$ & 44 & -76 & 12 & Lateral Occipital Cortex \\
2 & $5 / 55$ & 40 & -58 & -8 & Lateral Occipital Cortex \\
2 & $5 / 54$ & 44 & -74 & 20 & Lateral Occipital Cortex \\
2 & $5 / 49$ & 40 & -64 & 14 & Lateral Occipital Cortex \\
2 & $5 / 4$ & 50 & -60 & 6 & Medial Temporal Gyrus \\
2 & $5 / 25$ & 32 & -82 & 18 & Lateral Occipital Cortex \\
& $4 / 09$ & 50 & 0 & 44 & Medial Frontal Gyrus \\
1 & $3 / 87$ & 46 & 2 & 38 & Medial Frontal Gyrus \\
1 & $3 / 79$ & 40 & 10 & 24 & Medial Frontal Gyrus \\
1 & $3 / 53$ & 48 & 16 & 22 & Medial Frontal Gyrus \\
1 & $3 / 51$ & 52 & 4 & 48 & Medial Frontal Gyrus \\
1 & $3 / 49$ & 28 & -8 & 12 & Right Putamen \\
\hline
\end{tabular}

The results of brain activations in the EFT >Episodic memory contrast- cannabis users' average activations

Each activation pattern is reported by the number of clusters, number of voxels in each cluster, maximum z-value of the cluster, and the coordinates $(x, y, z)$ of the voxel with the highest $z$-value in the cluster. The reported clusters survive a whole-brain threshold of $Z>2.3(P<0.05)$. 


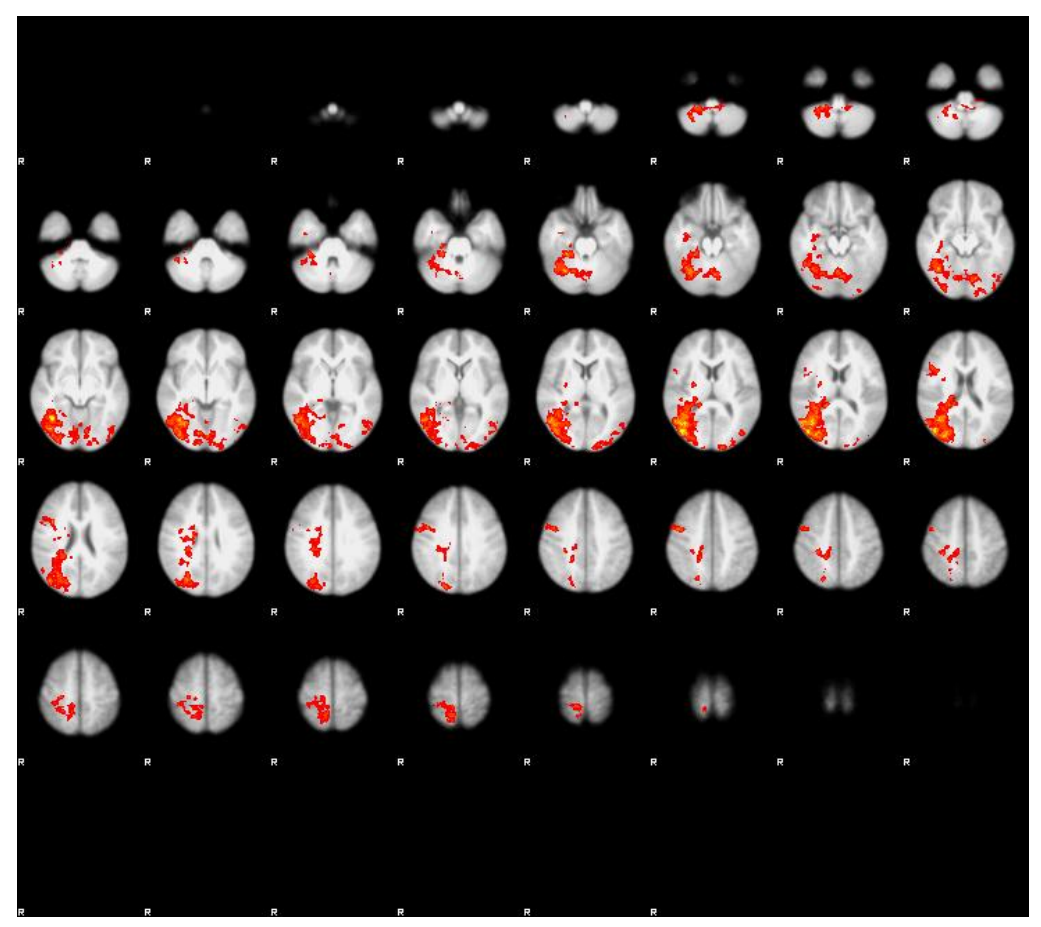

Brain activity patterns associated with EFT $>$ EM contrast cannabis users (Z-threshold $=>2.3$ )

c) Controls > cannabis users

\begin{tabular}{|c|c|c|c|c|c|}
\hline Cluster & Z-max & $\mathrm{X}$ & $\mathrm{Y}$ & $\mathrm{Z}$ & Brain region \\
\hline 2 & $3 / 42$ & -60 & -4 & -8 & Medial Temporal Gyrus \\
\hline 2 & $3 / 39$ & -44 & -16 & -14 & Superior Temporal Gyrus \\
\hline 2 & $3 / 34$ & -50 & -16 & -12 & Medial Temporal Gyrus \\
\hline 2 & $3 / 27$ & -54 & -24 & 0 & Superior Temporal Gyrus \\
\hline 2 & $3 / 25$ & -64 & -20 & -8 & Medial Temporal Gyrus \\
\hline 2 & $3 / 23$ & -50 & -18 & -8 & Medial Temporal Gyrus \\
\hline 1 & $4 / 73$ & -14 & -90 & -26 & Occipital Fusiform Gyrus \\
\hline 1 & $3 / 54$ & -22 & -84 & -24 & Occipital Fusiform Gyrus \\
\hline 1 & $3 / 46$ & -36 & -58 & -30 & Lateral Occipital Cortex \\
\hline 1 & $3 / 37$ & -16 & -74 & -32 & Cerebellum \\
\hline 1 & $3 / 32$ & -36 & -72 & -28 & Cerebellum \\
\hline 1 & $3 / 27$ & -20 & -74 & -32 & Cerebellum \\
\hline
\end{tabular}

The results of brain activations in the EFT $>$ Episodic memory contrast- Contols $>$ cannabis users

Each activation pattern is reported by the number of clusters, number of voxels in each cluster, maximum z-value of the cluster, and the coordinates $(x, y, z)$ of the voxel with the highest $z$-value in the cluster. The reported clusters survive a whole-brain

threshold of $Z>2.3(P<0.05$ 


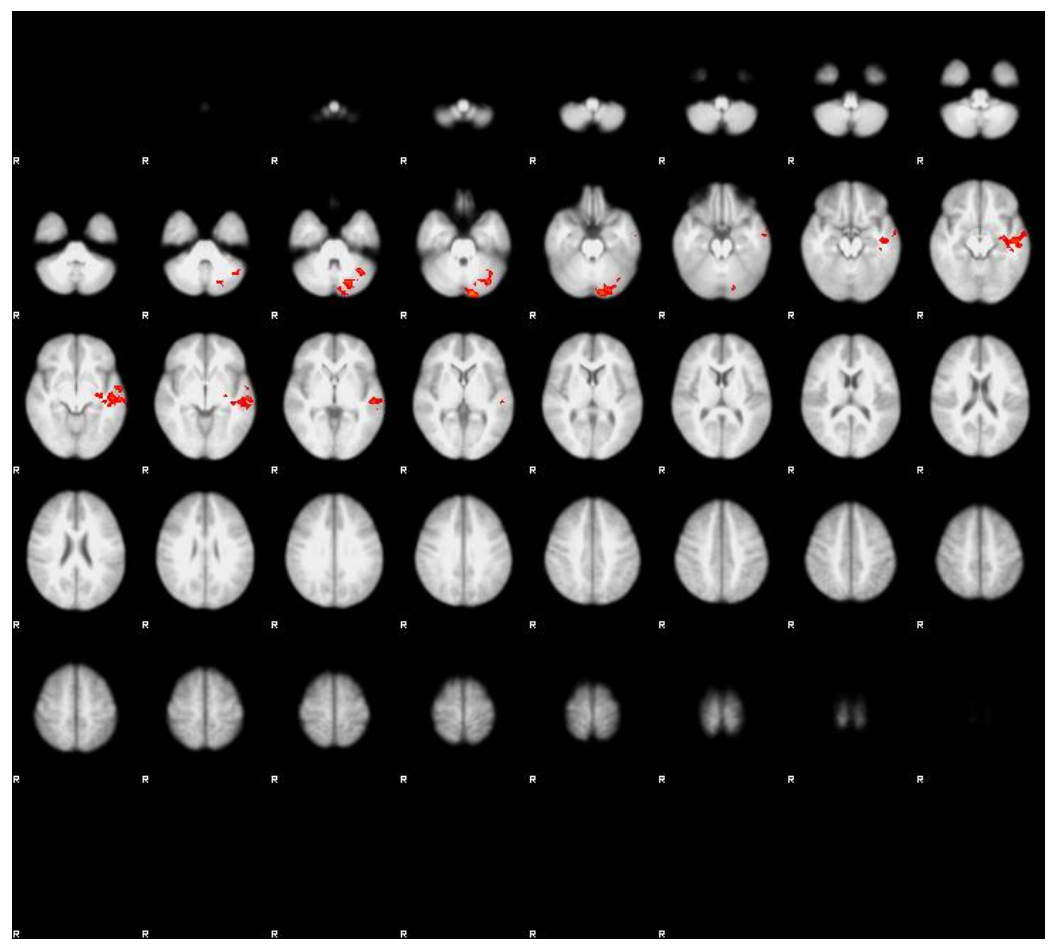

Brain activity differences associated with EFT $>$ EM contrast in control $>$ cannabis users $($ Z-threshold $=>2.3$ )

3) Contrast 3-Episodic memory (past)

a) Controls:

\begin{tabular}{|c|c|c|c|c|c|}
\hline Cluster & Z-max & $\mathrm{X}$ & $\mathrm{Y}$ & $\mathrm{Z}$ & Brain region \\
\hline 3 & $9 / 89$ & 32 & -74 & -50 & Cerebellum \\
\hline 3 & $9 / 31$ & 16 & -80 & -18 & Occipital Fusiform Gyrus, Lingual Gyrus \\
\hline 3 & $8 / 16$ & 34 & -76 & -46 & Cerebellum \\
\hline 3 & $7 / 84$ & 36 & -72 & -46 & Cerebellum \\
\hline 3 & $7 / 35$ & 20 & -34 & -12 & Right Hippocampus, Parahippocampal Gyrus \\
\hline 3 & $7 / 11$ & 30 & -62 & -44 & Cerebellum \\
\hline 2 & $6 / 46$ & -38 & -72 & 28 & Lateral Occipital Cortex \\
\hline 2 & $5 / 26$ & -42 & -74 & 30 & Lateral Occipital Cortex \\
\hline 2 & $4 / 85$ & -34 & -76 & 32 & Lateral Occipital Cortex \\
\hline 2 & $4 / 65$ & -44 & -32 & 44 & Postcentral Gyrus, Supramarginal Gyrus \\
\hline 2 & $4 / 62$ & -34 & -56 & 44 & Superior Parietal lobule, Angular Gyrus \\
\hline 2 & $4 / 6$ & -36 & -72 & 36 & Lateral Occipital Cortex \\
\hline 1 & $4 / 23$ & -56 & -48 & -4 & Medial Temporal Gyrus \\
\hline 1 & $4 / 18$ & -66 & -46 & 0 & Medial Temporal Gyrus, Angular Gyrus \\
\hline 1 & $3 / 81$ & -54 & -46 & -16 & Inferior temporal Gyrus \\
\hline 1 & $3 / 69$ & -52 & -48 & -10 & Inferior temporal Gyrus \\
\hline 1 & $3 / 41$ & -52 & -42 & -14 & Medial Temporal Gyrus \\
\hline 1 & $3 / 38$ & -62 & -30 & -6 & Medial Temporal Gyrus \\
\hline
\end{tabular}

The results of brain activations in the Episodic memory contrast- controls' average activations

Each activation pattern is reported by the number of clusters, number of voxels in each cluster, maximum z-value of the cluster, and the coordinates $(x, y, z)$ of the voxel with the highest $z$-value in the cluster. The reported clusters survive a whole-brain threshold of $Z>2.3(P<0.05)$. 


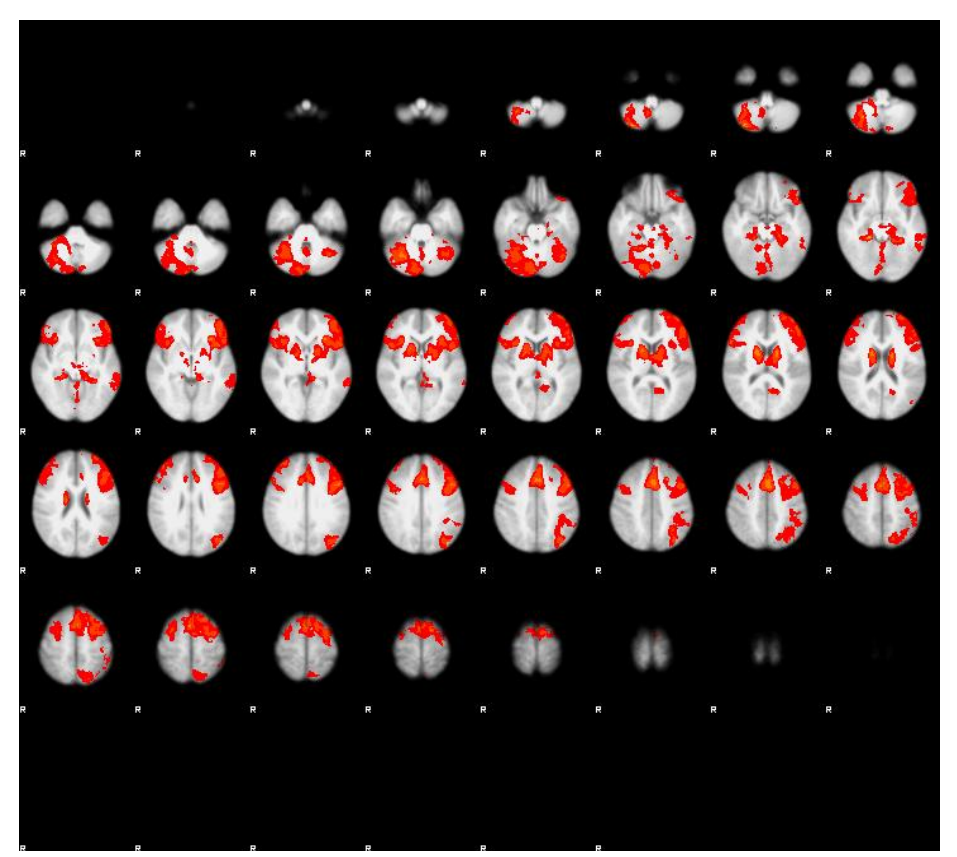

Brain activity patterns associated with episodic memory condition in drug-naïve controls $(Z$-threshold= $=2.3$ )

b) Cannabis users:

\begin{tabular}{|c|c|c|c|c|c|}
\hline Cluster & Z-max & $\mathrm{X}$ & $\mathrm{Y}$ & $\mathrm{Z}$ & Brain region \\
\hline 5 & $7 / 78$ & -48 & -40 & 48 & Postcentral Gyrus \\
\hline 5 & $7 / 51$ & -26 & 68 & 8 & Inferior Frontal Gyrus \\
\hline 5 & $6 / 65$ & 46 & -72 & -32 & Cerebellum \\
\hline 5 & $6 / 58$ & 0 & 26 & 46 & Paracingulate Gyrus \\
\hline 5 & $6 / 54$ & 46 & -64 & -42 & Cerebellum \\
\hline 5 & $6 / 46$ & 8 & -84 & -24 & Occipital Fusiform Gyrus \\
\hline 4 & $5 / 23$ & 50 & 20 & 32 & Medial Frontal Gyrus \\
\hline 4 & $5 / 12$ & 26 & -4 & 36 & Medial Frontal Gyrus \\
\hline 4 & $4 / 79$ & 34 & 20 & 32 & Medial Frontal Gyrus \\
\hline 4 & $4 / 48$ & 30 & 36 & 32 & Medial Frontal Gyrus \\
\hline 4 & $4 / 34$ & 36 & 24 & 34 & Medial Frontal Gyrus \\
\hline 4 & $3 / 88$ & 46 & 14 & 32 & Medial Frontal Gyrus \\
\hline 3 & $5 / 36$ & -66 & -40 & -10 & Medial Temporal Gyrus, Superior Temporal Gyrus \\
\hline 3 & $5 / 11$ & -60 & -44 & -10 & Medial Temporal Gyrus, Superior Temporal Gyrus \\
\hline 3 & $5 / 05$ & -64 & -38 & -6 & Medial Temporal Gyrus, Superior Temporal Gyrus \\
\hline 3 & $4 / 97$ & -64 & -40 & -14 & Medial Temporal Gyrus, Superior Temporal Gyrus \\
\hline 3 & $4 / 45$ & -66 & -30 & -10 & Medial Temporal Gyrus, Superior Temporal Gyrus \\
\hline 3 & $4 / 4$ & -66 & -28 & -12 & Medial Temporal Gyrus, Superior Temporal Gyrus \\
\hline 2 & $5 / 03$ & 34 & 26 & 4 & Insular cortex \\
\hline 2 & $4 / 95$ & 56 & 18 & 0 & Orbitofrontal Gyrus Inferior Frontal Gyrus \\
\hline 2 & $4 / 39$ & 44 & 28 & 0 & Orbitofrontal Gyrus Inferior Frontal Gyrus \\
\hline 2 & $4 / 26$ & 38 & 16 & 6 & Orbitofrontal Gyrus Inferior Frontal Gyrus \\
\hline 2 & $3 / 78$ & 30 & 20 & -6 & Insular cortex \\
\hline 2 & $3 / 56$ & 38 & 26 & 0 & Frontal Operculum Cortex \\
\hline 1 & $4 / 65$ & -32 & -38 & -30 & Occipital Fusiform Gyrus \\
\hline 1 & $4 / 47$ & -42 & -30 & -26 & Occipital Fusiform Gyrus \\
\hline 1 & $4 / 36$ & -42 & -34 & -28 & Superior Temporal Gyrus \\
\hline 1 & $3 / 84$ & -30 & -68 & -24 & Superior Temporal Gyrus \\
\hline 1 & $3 / 54$ & -36 & -34 & -22 & Parahippocampal Gyrus \\
\hline 1 & $3 / 24$ & -30 & -60 & -24 & Parahippocampal Gyrus \\
\hline
\end{tabular}

The results of brain activations in the Episodic memory contrast- cannabis users' average activations 
Each activation pattern is reported by the number of clusters, number of voxels in each cluster, maximum z-value of the cluster, and the coordinates $(x, y, z)$ of the voxel with the highest $z$-value in the cluster. The reported clusters survive a whole-brain threshold of $Z>2.3(P<0.05)$.

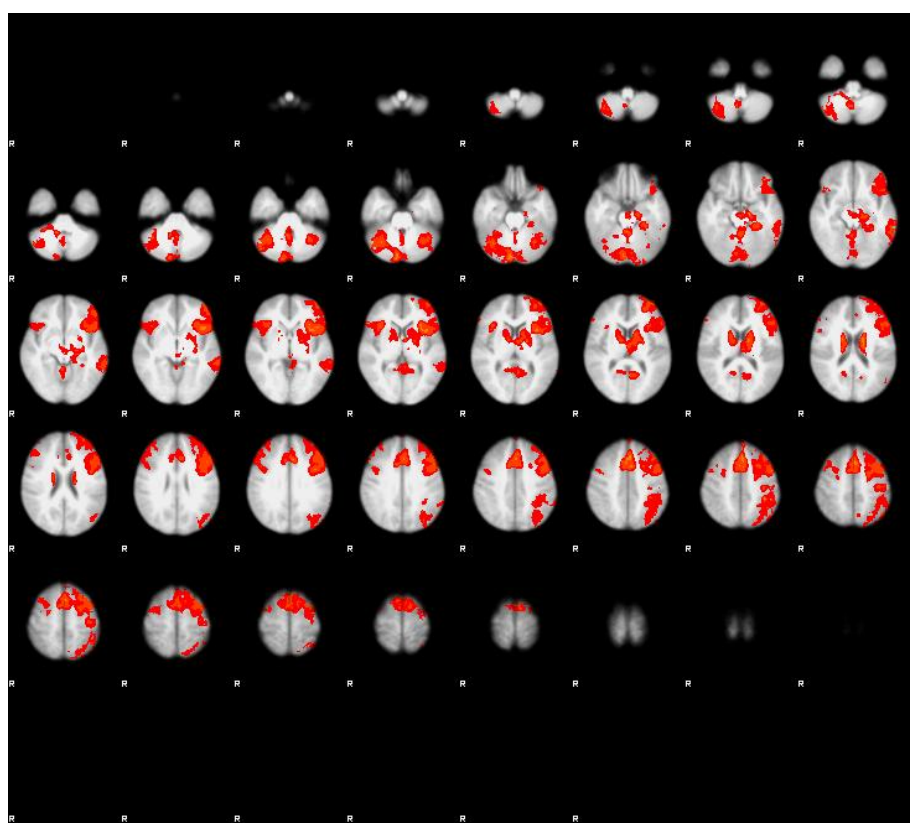

Brain activity patterns associated with episodic memory condition contrast in cannabis users (Z-threshold= >2.3)

c) Controls $>$ cannabis users

\begin{tabular}{cccccc} 
Cluster & Z-max & X & Y & Z & Brain region \\
\hline 1 & $3 / 55$ & 48 & 48 & 8 & Right Frontal pole \\
1 & $3 / 53$ & 46 & 54 & 8 & Right Frontal pole \\
1 & $3 / 31$ & 48 & 42 & 6 & Inferior Frontal Gyrus \\
1 & $3 / 21$ & 5 & 42 & 0 & Inferior Frontal Gyrus \\
1 & $3 / 2$ & 46 & 40 & 0 & Inferior Frontal Gyrus \\
1 & $3 / 19$ & 42 & 54 & 16 & Right Frontal pole \\
\hline
\end{tabular}

The results of brain activations in the Episodic memory contrast- Contols>cannabis users

Each activation pattern is reported by the number of clusters, number of voxels in each cluster, maximum z-value of the cluster, and the coordinates $(x, y, z)$ of the voxel with the highest $z$-value in the cluster. The reported clusters survive a whole-brain threshold of $Z>2.3(P<0.05$ 


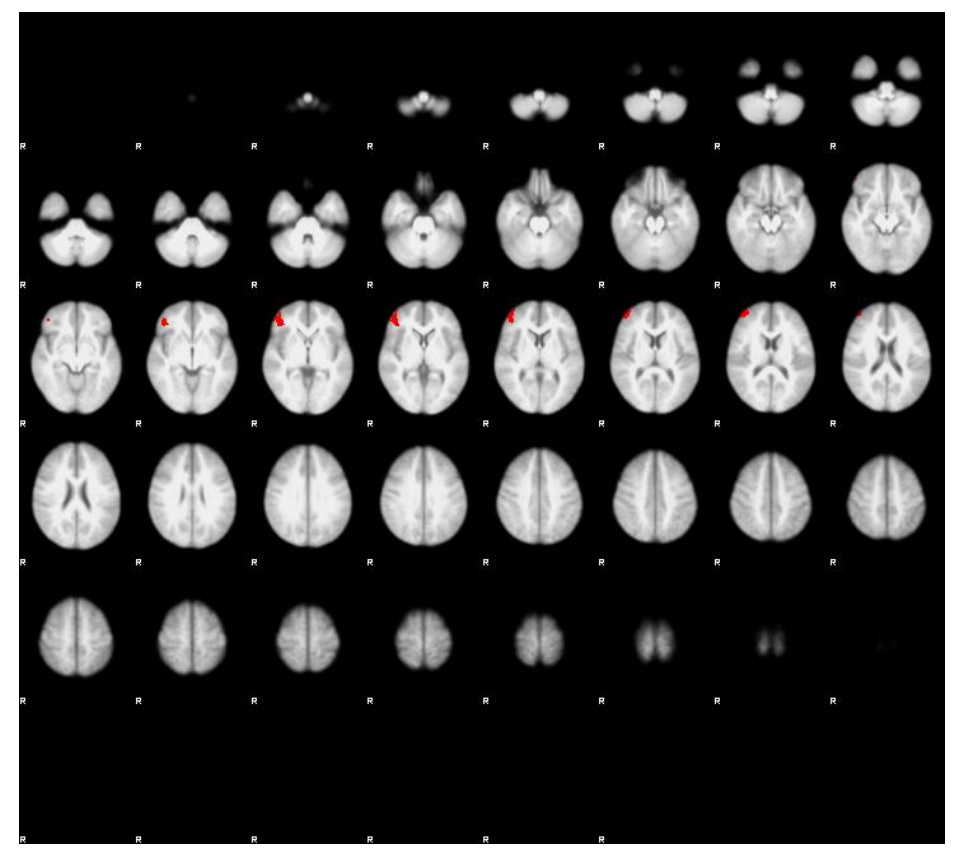

Brain activity differences associated with episodic memory condition in control $>$ cannabis users (Z-threshold $=>2.3$ ) 